\title{
Regulation of the Unfolded Protein Response by BiP AMPylation protects photoreceptors from light-dependent degeneration.
}

Andrew T. Moehlman ${ }^{1}$, Amanda K. Casey ${ }^{2}$, Kelly Servage ${ }^{2,3}$, Kim Orth $^{2,3^{*}}$, Helmut Krämer ${ }^{1,4^{*}}$

\section{Affiliations:}

${ }^{1}$ Department of Neuroscience, UT Southwestern Medical Center, Dallas, TX.

$9 \quad{ }^{2}$ Department of Molecular Biology UT Southwestern Medical Center, Dallas, TX.

$10{ }^{3}$ Department of Biochemistry, UT Southwestern Medical Center, Dallas, TX and Howard

11 Hughes Medical Institute, Dallas, TX.

$12{ }^{4}$ Department of Cell Biology, UT Southwestern Medical Center, Dallas, TX.

$14 *$ Correspondence to:

$15 \quad$ Helmut.Kramer@,UTSouthwestern.edu,

$16 \quad \underline{\text { Kim.Orth@utsouthwestern.edu }}$ 


\section{Abstract}

In response to environmental, developmental, and pathological stressors, cells engage

homeostatic pathways to maintain their function. Among these pathways, the Unfolded Protein major ER chaperone and regulator of the Unfolded Protein Response. This work elucidates the importance of the reversible AMPylation of BiP in maintaining the Drosophila visual system in response to stress. After 72 hours of constant light, photoreceptors of fic-null and AMPylationresistant $\mathrm{BiP}^{\mathrm{T} 366 \mathrm{~A}}$ mutants, but not wild-type flies, display loss of synaptic function, disintegration of rhabdomeres, and excessive activation of ER stress reporters. Strikingly, this phenotype is reversible: photoreceptors regain their structure and function within 72 hours once returned to a standard light:dark cycle. These findings show that Fic-mediated AMPylation of $\mathrm{BiP}$ is required for neurons to adapt to transient stress demands.

\section{Introduction}

Post-translational modifications (PTMs) of proteins are important for rapid responses to environmental challenges of cells. One such PTM is AMPylation, the reversible addition of 40 2018; Harms et al., 2016). Eukaryotic Fic, an ER-resident type-II membrane protein (Rahman et adenosine monophosphate (AMP) to hydroxyl groups (also known as adenylylation) (Brown et al., 1971; Casey \& Orth, 2018; Kingdon et al., 1967; Woolery et al.). AMPylation is catalyzed by at least two protein families, among them the conserved Fic-domain proteins (Casey \& Orth, al., 2012), AMPylates BiP (GRP78), a highly conserved and ubiquitous ER chaperone (Ham et 
al., 2014; Preissler et al., 2015). Working together with a multitude of associated quality control proteins, $\mathrm{BiP}$ is critical for the translocation, folding, and secretion of proteins from the ER as well as for aiding in the clearing of misfolded ER aggregates and degradation of membraneassociated proteins (Hendershot et al., 1988; Kozutsumi et al., 1988; Meunier et al., 2002). BiP is both a mediator and transcriptional target of the Unfolded Protein Response (UPR), a coordinated cell signaling pathway that is activated during times of high misfolded protein levels in the ER. Like many protein chaperones, BiP depends on its ATPase activity to undergo a conformational change to bind to its substrates (Gaut \& Hendershot, 1993). AMPylation locks BiP into a state resembling the ATP-bound conformation with high substrate off-rates, thereby inhibiting its chaperone function (Preissler, Rohland, et al., 2017; Wieteska et al., 2017).

In agreement with this PTM's inhibitory role, BiP AMPylation levels are linked to protein homeostasis (Ham et al., 2014). Reduction of ER protein load promotes Fic-mediated AMPylation of BiP, whereas Fic catalyzes the deAMPylation of BiP under elevated ER stress conditions (Ham et al., 2014; Preissler et al., 2015). This switch in Fic's activity is linked to a key regulatory salt bridge in eukaryotic Fic. Mutations in Fic that disrupt this salt bridge result in an overactive AMPylator that lacks deAMPylation activity (Casey et al., 2017; Preissler, Rato, et al., 2017). Together, these studies suggest a model in which BiP is AMPylated in times of low ER stress, creating a reserve pool of inactive BiP that can be readily activated to respond to changes of ER homeostasis (Figure 1A). This reserve pool of BiP is proposed to act as a buffer to attenuate or shorten the need for a more dramatic activation of the transcriptional and translational arms of the UPR (Casey et al., 2017; Preissler, Rato, et al., 2017; Wieteska et al., 2017). However, the physiological importance of endogenous Fic-mediated AMPylation for remains unclear. 
In the fruit fly, Drosophila melanogaster, we previously demonstrated that fic-null mutants harbor a defect in visual signaling, as assessed by electroretinogram (ERG). The wellcharacterized Drosophila visual system has proven a valuable model for many fields, such as neuroscience (Borycz et al., 2002; Sugie et al., 2015), cell signaling (Dolph et al., 1993; Scott et al., 1995), protein trafficking (Akbar et al., 2009; Lee et al., 2003), and neurodegeneration (Johnson et al., 2002; Leonard et al., 1992; Ryoo et al., 2007). The specialized photoreceptor cells possess tightly packed microvilli-like membranes, termed rhabdomeres, that endow remarkable sensitivity to minute changes in light conditions (Montell, 2012). The ability to maintain this sensitivity is critical for flight behavior, foraging, and escape from predators. Thus, under a wide range of conditions, photoreceptors must maintain their light detection cascade, which requires the constant production, trafficking, and degradation of proteins through the endomembrane system (Colley et al., 1995; Kiselev et al., 2000; Rosenbaum et al., 2006).

We postulated that as a regulator of proteostasis and the UPR, BiP must be tightly regulated for proper maintenance of vision in the fly. Here we demonstrate that mutants lacking AMPylation of BiP at a specific residue, Thr366, possess the same ERG defect as fic-null animals, implicating BiP as the target of Fic necessary for visual signaling. We go on to find that application of an eye-specific stress, constant light, leads to exaggerated electrophysiology defects and the loss of photoreceptor rhabdomeres, akin to neurodegeneration. However, these defects exhibit a remarkable and unique reversibility: structural and functional phenotypes observed in light-stressed fic-null and AMPylation-resistant $B i P^{\mathrm{T} 366 \mathrm{~A}}$ mutants are reversed after the flies are returned to a standard light/dark cycle. In addition, we identify changes in the regulation of UPR during constant light stress in these mutants, implicating ER dysregulation as the cause of the inability to adapt to altered light conditions. 


\section{Results}

\section{$\mathrm{BiP}^{\mathrm{T} 366 \mathrm{~A}}$ rescues over-expression of constitutively active AMPylating Fic ${ }^{\mathrm{E247G}}$}

To test the hypothesis that BiP is a critical target of Fic AMPylation in vivo (Figure 1A), we designed and generated transgenes expressing wild-type and AMPylation-resistant FLAGtagged $\mathrm{BiP}$ proteins under control of the endogenous BiP promoter (Figure 1- figure

supplement 1A). BiP null fly mutants die early in development; this lethality is rescued by including a copy of the genomic transgene expressing FLAG-BiP ${ }^{\mathrm{WT}}$ or the AMPylation-resistant FLAG-BiP ${ }^{\mathrm{T} 366 \mathrm{~A}}$ mutant (Figure 1B). We will refer to these rescued flies as $B i P^{\mathrm{WT}}$ or $B i P^{\mathrm{T} 366 \mathrm{~A}}$, respectively. In contrast, a BiP transgene mutated at a second reported AMPylation site (Casey et al., 2017; Preissler et al., 2015), BiP ${ }^{\mathrm{T} 518 \mathrm{~A}}$, did not rescue $\mathrm{Bi} \mathrm{P}^{-/-}$lethality. As fic null mutants that lack BiP AMPylation are viable, the lethality of the $B i P^{\mathrm{T} 518 \mathrm{~A}}$ mutant is not likely to be due to the loss of AMPylation (Casey et al., 2017). Instead, these observations indicate an essential role for $\mathrm{Thr}^{518}$ in BiP's chaperone activity. Consistent with this notion, the equivalent residue, Thr ${ }^{538}$, in the $S$. cerevisiae BiP homolog Kar2 is required for survival under heat stress even though yeast lack both Fic domain proteins and BiP AMPylation (Figure 1- figure supplement 1B).

Previously, we reported that over-expression of the constitutively active AMPylating $\mathrm{Fic}^{\mathrm{E} 247 \mathrm{G}}$ was lethal in a fic-null fly background $\left(f i c^{30 \mathrm{C}}\right)$ because it lacks the essential deAMPylation activity (Casey et al., 2017). We tested whether flies expressing the AMPylationresistant $\mathrm{BiP}^{\mathrm{T} 366 \mathrm{~A}}$ could survive this lethality. Consistent with previous findings, we observe over-expression of the mutant UAS-Fic ${ }^{\mathrm{E} 247 \mathrm{G}}$ transgene using the ubiquitous $D a$-Gal4 driver was lethal in an otherwise fic-null animal (Figure 1C). Lethality of the constitutively active AMPylating Fic ${ }^{\mathrm{E} 247 \mathrm{G}}$ was suppressed in flies expressing the AMPylation-resistant BiP ${ }^{\mathrm{T} 366 \mathrm{~A}}$ but 
111 not BiP ${ }^{\mathrm{WT}}$ (Figure 1C). These results indicate that $\mathrm{Thr}^{366}$ of $\mathrm{BiP}$ is a physiologically relevant

$112 \quad$ AMPylation target in vivo.

\section{The UPR protects eyes from overactive AMPylation}

To test the interaction between Fic-mediated AMPylation and the UPR, we employed an eye-specific Fic gain-of-function model. Eye-specific expression of the constitutively active background, eye-specific expression of constitutively active AMPylating Fic ${ }^{\mathrm{E} 24 \mathrm{G}}$ yields only a mildly rough eye (Figure $\mathbf{2 A}$ ). We used this intermediate phenotype to assess genetic interactions between Fic $^{\mathrm{E} 247 \mathrm{G}}$ and components of the UPR with a candidate-based targeted RNAi screen (Table 1). Fic ${ }^{\mathrm{E} 247 \mathrm{G}}$-induced eye roughness was significantly enhanced by knockdown of the key UPR components Perk, Atf4, and Irel (Figure 2C-E and Table 1), but not ATF6 (Figure 2B). Furthermore, $X b p l$ knockdown in conjunction with Fic ${ }^{\mathrm{E} 247 \mathrm{G}}$ expression was lethal (Figure 2F). By contrast, knockdown of these UPR genes in in the absence of Fic ${ }^{\mathrm{E} 247 \mathrm{G}}$ did not influence eye phenotype or fly survival (Figure 2A'-F'). These genetic interactions suggest a role for UPR signaling in mitigating cellular stress imposed by expressing the constitutively active AMPylating Fic ${ }^{\mathrm{E} 247 \mathrm{G}}$ in the eye.

\section{AMPylation of $\mathrm{BiP}$ is necessary for maintaining vision}

The findings that BiP is a target of Fic in vivo and that silencing UPR pathway components enhances the severity of the constitutively active AMPylating Fic ${ }^{\mathrm{E} 247 \mathrm{G}}$-induced rough-eye phenotype prompted us to assay the physiological effects of cellular stress in flies 
lacking BiP AMPylation. To do this we utilized flies that are either null for $f i c\left(f i c^{30 C}\right)$ or express the AMPylation-resistant BiP ${ }^{\mathrm{T} 366 \mathrm{~A}}$ instead of wild-type BiP. By using this strategy, we are able to discern BiP AMPylation-specific changes from other potential changes that are due to as-yetunknown targets of Fic AMPylation.

As previously reported in ERG recordings, fic-null flies display a reduction of the initial response (termed the ON Transient, Figure 3A) to a light pulse compared to wild-type controls. Interestingly, $B i P^{\mathrm{T} 366 \mathrm{~A}}$, but not $B i P^{\mathrm{WT}}$ flies, exhibited the same defect in ON Transients as $f i c^{30 \mathrm{C}}$ mutants, consistent with BiP being the primary target of Fic AMPylation required for proper visual neurotransmission (Figure 1- figure supplement 2). Of note, we used an eye-specific RNAi construct against white to minimize any effect of the mini-white gene used as a marker in these BiP transgenes. When we compared ERG traces of $f i c^{30 \mathrm{C}}$ and $B i P^{\mathrm{T} 366 \mathrm{~A}}$ flies in white+ (red eyed) backgrounds, the reductions in ON transients were no longer detectable (Figure 1- figure

supplement 3). This is likely due to the previously established protective effect provided by the red pigment in white ${ }^{+}$flies. Indeed, a similar white-dependent phenotype has been reported for photoreceptor synaptic plasticity after prolonged light exposure (Damulewicz et al., 2017; Sugie et al., 2015), consistent with previous observations that a functional white gene masks some degenerative phenotypes in the retina (Lee \& Montell, 2004; Soukup et al., 2013). Therefore, we used the white-eyed flies (referred to as $w^{1118}$ ) to test whether AMPylation may play a role in this type of photoreceptor plasticity, which is induced by rearing flies in uninterrupted low light for 72 hours (Damulewicz et al., 2017; Sugie et al., 2015).

We conducted ERG recordings under different light conditions with four fly lines, $w^{1118}$, $f i c^{30 \mathrm{C}}, B i P^{\mathrm{WT}}$ and $B i P^{\mathrm{T} 366 \mathrm{~A}}$ (Figure 3B). Compared to age-matched siblings reared under the standard $12 \mathrm{hr}$ Light:12 hr Dark (LD) treatment, $f i c^{30 \mathrm{C}}$ and BiP $P^{\mathrm{T} 366 \mathrm{~A}}$ flies reared for three days 
under continuous light (LL) at 500 lux exhibited severe ERG defects. This included reduction in the sustained negative potential (SNP), a measure of photoreceptor activation, and loss of ON and OFF transients, which reflect synaptic transmission to downstream L1/L2 lamina neurons (Figure 3C \& D). Wild-type controls maintained healthy OFF transients following LL, but ON transients were reduced, reflecting the sensitivity of this component to various light conditions (Figure 3D). To test for behavioral consequences, we assayed $w^{1118}$ and $f i c^{30 \mathrm{C}}$ flies after 72 hours of LD or LL treatment for light-induced startle behavior using single-fly activity chambers (Ni et al., 2017). Following a 5-min light pulse, LD-reared $f i c^{30 \mathrm{C}}$ flies exhibited a startle response indistinguishable from control $w^{1118}$ flies, whereas $f i c^{30 C}$ flies, but not $w^{1118}$ flies, failed to respond to the light pulse after 72 hours of LL (Figure 3- figure supplement 1). Thus, Ficmediated AMPylation is required to maintain vision acuity under LL conditions.

We next designed experiments to test whether these functional ERG changes in flies lacking AMPylation reflected light-induced neurodegeneration or a failure to adapt to constant stimulation. First, we asked if the LL-induced ERG defects of $B i P^{\mathrm{T} 366 \mathrm{~A}}$ and $f i c^{30 \mathrm{C}}$ flies were reversible. We reared mutant and control flies for 72 hours in LL followed by 72 hours of recovery in LD (referred to as "Rec"; Figure 3B). This recovery period was sufficient to restore both healthy OFF transients and SNPs in $B i P^{\mathrm{T} 366 \mathrm{~A}}$ and $f i c^{30 \mathrm{C}}$ flies (Figure 3C \& D). Second, we asked if the intensity of the light would exaggerate the defects of $B i P^{\mathrm{T} 366 \mathrm{~A}}$ and $f i c^{30 \mathrm{C}}$ flies. Exposure of mutant or control flies with 5000 lux, instead of 500 lux, did not alter the severity of ERG defects, indicating the changes were not simply a reflection of the increased amount of total light exposure during LL treatment (Figure 3- figure supplement 2). Third, we asked if prolonging the LL stress would alter the reversibility of these defects. Mutant flies reared under LL for ten days retained the capability to recover healthy ERG traces after only three days on 
180 LD, indicating that photoreceptors are not dying but maintained during prolonged light stress

181 (Figure 3- figure supplement 3). Together, these data support a model for a dysregulated

182 adaptive response, rather than phototoxicity, inducing the reversible loss of OFF transients and

183 reduced SNPs.

189 However, following 72 hours of LL (500 lux), fic ${ }^{30 \mathrm{C}}$ and BiP $P^{\mathrm{T} 366 \mathrm{~A}}$ mutants, but not $w^{1118}$ and

$190 B i P^{\mathrm{WT}}$ controls, displayed severe defects in the integrity of rhabdomeres, the microvilli-like

191 membrane structures that house the phototransduction cascade (Figure 4B). After a three-day

192 recovery at $\mathrm{LD}$, the rhabdomeres were nearly restored in both AMPylation-deficient mutants

193 (Figure 4C). To quantify these structural changes in large cohorts of flies, we assessed flies for

194 the presence of wild-type "deep pseudopupils" (DPP) (Figure 4D). Visualization of the DPP

195 affords an assessment of rhabdomere structural integrity in living flies (Franceschini \&

196 Kirschfeld, 1971). Consistent with the TEM data, 3 days of LL caused loss of DPP in $f i c^{30 C}$ and

$197 B P^{\mathrm{T} 366 \mathrm{~A}}$, and DPPs returned after a 3-day recovery (Figure 4D). This suggests that proper

198 regulation of BiP through AMPylation is required for maintaining both function and structure of 199 photoreceptor cells. 


\section{Fic regulates ER stress signaling in the retina and lamina neuropil}

Given the unique role of Fic in both AMPylating and deAMPylating BiP to modulate its chaperone activity and maintaining ER homeostasis, we evaluated $f i c^{30 \mathrm{C}}$ flies for changes in the UPR under LD, LL, and Rec conditions. First, we performed immunohistochemistry against BiP, a transcriptional target of the UPR, which is upregulated during states of ER stress (Gardner et al., 2013; Ham et al., 2014). After 3 days of LL, sections of $f i c^{30 \mathrm{C}}$ revealed increased levels of $\mathrm{BiP}$ in retinas and in the lamina neuropils where photoreceptor axons form synapses onto lamina neurons. BiP levels returned to control levels following three days of recovery (Figure 5A \& B). To further assess UPR signaling in these tissues, we utilized a sensor for Ire1-mediated Xbp1 splicing (Sone et al., 2013) and an Atf4 translational reporter which serves as a proxy for Perkmediated phosphorylation of eIF-2a (Kang et al., 2015). In wild-type flies, Xbp1-GFP was slightly induced in the lamina after 24 hours of LL in wild-type flies and the signal decreased over time (Figure 5C, top row, \& 5D). However, in ic $^{30 \mathrm{C}}$ flies, the Xbp1-GFP signal in the lamina continued to increase after 48 hours of LL and remained elevated after 72 hours (Figure bottom row, \& 5D). In the retina, control flies showed little to no increase of Xbp1-GFP levels, while $f i c^{30 \mathrm{C}}$ flies showed a significant transient increase after one and two days LL. With the Atf4-DsRed reporter, we observed a significant increase of signal in both the retina and lamina of wild-type flies after one day, but no difference in $f i c^{30 C}$ mutants at one or two days LL when compared to LD controls (Figure 5E \& F). However, by three days of LL, Atf4-DsRed reporter activity in the wild-type flies returned to control levels, while the $f i c^{30 \mathrm{C}}$ mutants showed a significant increase in both the retina and lamina neuropil (Figure $5 \mathbf{E} \boldsymbol{\&} \mathbf{F}$ ). These changes in UPR signaling were reversible as each of the reporters returned to near control intensity after 72 hours of LD recovery (Figure 5A, $\mathbf{C} \boldsymbol{\&} \mathbf{E}$, last columns). The elevated UPR response in 
$224 \mathrm{fic}^{30 \mathrm{C}}$ mutants correlated with the timing of the observed severe defects in the integrity of

225 rhabdomeres (Figure 4B). Together, these data identify a crucial role for Fic-mediated BiP

226 AMPylation in regulating UPR signaling during homeostatic responses of photoreceptor

227 neurons.

Discussion

Here we demonstrate that $\mathrm{BiP}$ is a critical in-vivo target of Fic-mediated AMPylation, as an AMPylation-resistant BiP blocks over-expressed constitutively active AMPylating Fic ${ }^{\mathrm{E} 247 \mathrm{G}}$ lethality and recapitulates fic-null vision defects. This work also sheds light on a novel physiological role for AMPylation/deAMPylation control of BiP: fine-tuning UPR signaling to allow for visual system adaptation. We observe genetic interactions with the constitutively active AMPylating Fic ${ }^{\mathrm{E} 247 \mathrm{G}}$ and the UPR sensors Ire1 and PERK as well as their effectors, perhaps due to the critical role of $\mathrm{BiP}$ as both a regulator (Amin-Wetzel et al., 2017; Bertolotti et al., 2000; al., 2014; Kozutsumi et al., 1988). Indeed, we hypothesize that unregulated Fic ${ }^{\text {E247G }}$, in the absence of deAMPylation activity, AMPylates excess BiP, excluding it from its normal chaperone role and leading to cell death (Casey et al., 2017; Truttmann et al., 2017). In support suppressed by AMPylation-resistant $\mathrm{BiP}^{\mathrm{T} 366 \mathrm{~A}}$. Furthermore, the enhancement of the rough-eye Fic $^{\mathrm{E} 247 \mathrm{G}}$ phenotype by knockdown of the Ire1 and PERK pathways suggest a protective role for the UPR, perhaps through the known effects on translation by Ire1-mediated decay of mRNA, Xbp1-driven transcription or PERK-mediated phosphorylation of eIF-2a (Gardner et al., 2013). 
Our work supports an in-vivo requirement for Fic-mediated AMPylation of $\mathrm{BiP}^{\mathrm{T} 366}$ in the context of long-term adaptation to prolonged light exposure. $\mathrm{BiP}^{\mathrm{T} 366}$ replacement mutants phenocopy fic-null flies in both the light-induced blindness and the unexpected recovery from this phenotype. These functional changes are mirrored in the structural changes of photoreceptor rhabdomeres. Rhabdomere appearance of AMPylation deficient flies was reminiscent of retinal degeneration mutants (Ryoo et al.; Smith et al., 1991), however the remarkable recovery of the tissue structure in three days is unlike any reported retinal degeneration phenotype. Together, this work demonstrates a seminal role for Fic-mediated AMPylation of BiP in vivo: enabling photoreceptors to adapt and maintain both structural and functionality integrity during periods of prolonged stress due to uninterrupted light stimulation. The exact mechanism through which these defects in fic mutants arise remains undetermined, but previous studies have demonstrated a requirement for maintaining normal ER folding and trafficking of transmembrane visual signaling components, such as Rhodoposin (Colley et al., 1995; Rosenbaum et al., 2006). This intense demand for proper ER stress regulation sensitizes the eye to otherwise mild defects in fic mutants, and the additional demands posed by the stress of constant light stimulation.

We also observed that loss of BiP AMPylation deregulates, but does not block, the UPR to this physiological stress. This observation supports previous claims that AMPylation and deAMPylation of BiP aids in maintaining ER homeostasis (Figure 1A) by establishing a reserve pool of BiP that can readily be deAMPylated in response to acute ER insults (Casey et al., 2017; Ham et al., 2014; Preissler, Rato, et al., 2017). This regulation would allow for fine-tuning of the UPR response under specific contexts, comparable to findings in C. elegans in which fic- 1 and hsp3 (a BiP homologue) mutants are sensitive to bacterial infection (Truttmann et al., 2016). We speculate that the eye requires tight control of BiP activity and suppression of UPR signaling, to 
facilitate adaptation of the vision signaling cascade. Under standard LD conditions, only slight differences are observed, presumably because ER stress is low (Figure $5 C \& \mathbf{D}, \mathbf{1}^{\text {st }}$ column). However, exposure to constant light results in ER stress and UPR signaling, which wild-type flies can clear, presumably because there is a reserve pool of AMPylated BiP to rapidly respond to the stress. In fic mutants, we speculate, loss of the reserve BiP results in the UPR dysregulation revealed by the IreI and PERK activity reporters (Figure $\mathbf{5 C} \boldsymbol{\&} \mathbf{D})$ as a larger proportion of BiP would be previously engaged and not able to respond to the extra stress. in increased expression of UPR-regulated proteins while blocking production of the visual signaling components necessary for adapting to transient stress. affects the interactions of BiP with these proteins in vivo; however, in-vitro work suggests that AMPylation of BiP abolishes its inhibitory effect on Ire1 dimerization and activation (AminWetzel et al., 2017). We speculate that the loss of BiP AMPylation/deAMPylation cycle in a fic null affects both the ability of BiP to quickly respond to misfolded protein aggregates and to regulate UPR activation. Future studies on the dynamic role of reversible BiP AMPylation and 


\section{Materials and Methods}

\section{Fly stocks and genetics}

Bloomington Stock Center provided $w^{1118}(\mathrm{BS} \# 3605), B i P^{\mathrm{G} 0102} / F M 7 c$ (BS\#11815), Dawas previously described (Casey et al., 2017). Lines used in the RNAi screen are described in Table S1 and were obtained from Bloomington Stock Center and the Vienna Drosophila Resource Center (Dietzl et al., 2007). The Atf45'UtR_dsRed (Kang et al., 2015) and the Xbp1GFP (Sone et al., 2013) lines were a gift from Dr. Don Ryoo (NYU) and were recombined with the $f i c^{30 \mathrm{C}}$ allele. We generated the $\mathrm{p}\left[\text { gen3xFLAG-BiP }{ }^{\mathrm{WT}}\right]^{\mathrm{AttP}-89 \mathrm{E} 11}, \mathrm{p}\left[\text { gen3xFLAG-BiP }{ }^{\mathrm{T} 366 \mathrm{~A}}\right]^{\mathrm{AttP}-}$ ${ }^{89 \mathrm{E} 11}$, $\mathrm{p}\left[\mathrm{gen} 3 \mathrm{xFLAG}-\mathrm{BiP}^{\mathrm{T} 518 \mathrm{~A}}\right]^{\mathrm{AttP}-89 \mathrm{E} 11}$ and $\mathrm{p}\left[G M R\right.$-dsRNA $\left.{ }^{\text {white }}\right]$ alleles using the Phi30C integrase strategy (Venken et al., 2006). p[GMR-dsRNA $\left.{ }^{\text {white }}\right]$ was recombined with the $f i c^{30 C}$ allele and white-eyed candidates were screened for the fic allele by PCR. BiP $P^{\mathrm{G} 0102}$;; p[gen3xFLAG-BiP $\left.{ }^{\mathrm{WT}}\right]^{\mathrm{AttP}-89 \mathrm{E} 11}$ and BiP ${ }^{\mathrm{G} 0102} ;$;p[gen3xFLAG-BiP $\left.{ }^{\mathrm{T} 366 \mathrm{~A}}\right]^{\mathrm{AttP}-89 \mathrm{E} 11}$ stocks were made by crossing males harboring the genomic transgene to $B P^{\mathrm{G} 0102} / \mathrm{FM} 7 \mathrm{c}$ female flies. Surviving males were backcrossed to $B i P^{\mathrm{G} 0102} / \mathrm{FM} 7 \mathrm{c}$ female flies, and stable stocks were established from the resulting progeny. None of the rare escaping $B i P^{\mathrm{G} 0102} ; ; \mathrm{p}\left[\mathrm{gen} 3 \mathrm{xFLAG}-\mathrm{BiP}^{\mathrm{T} 518 \mathrm{~A}}\right]^{\mathrm{AttP}-89 \mathrm{E} 11}$ male flies were fertile. The LongGMR-Gal4,UAS ${ }^{\mathrm{Scer}}-\mathrm{V} 5-\mathrm{Fic}^{\mathrm{E} 247 \mathrm{G} \_ \text {attP-B3}} / \mathrm{TM} 6 \mathrm{~B}, \mathrm{Hu}$ and $D a-$

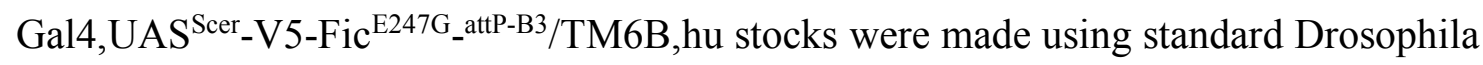
recombination and crossed into $w^{1118}$ and $w^{1118} ;$ fic $c^{30 C}$ backgrounds.

\section{List of flies strains and stocks used}

$$
w^{1118}(\mathrm{BS} \# 3605)
$$

OreR 
314

315

316

317

318

319

320

321

322

323

324

325

326

327

328

331

332

333

334
$; f i c^{30 \mathrm{C}}$

$w^{1118} ; f^{30 C}$

$w^{1118} ; \mathrm{p}\left[G M R\right.$-dsRNA $\left.{ }^{\text {white }}\right]$

$w^{1118} ; f_{i c}^{30 \mathrm{C}}, \mathrm{p}\left[\right.$ GMR-dsRNA $\left.{ }^{\text {white }}\right]$

$w^{1118} ;$ fic $^{30 \mathrm{C}} ;$ LongGMR-Ga14,UAS ${ }^{\text {Scer-V5-Fic }}{ }^{\mathrm{E} 247 \mathrm{G} \text { _ attP-B3}} / \mathrm{TM} 6 \mathrm{~B}, \mathrm{hu}$

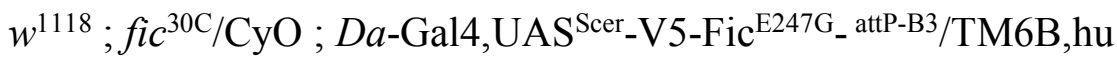

$B i P^{\mathrm{G} 0102} / \mathrm{FM} 7 \mathrm{c}(\mathrm{BS} \# 11815)$

$w^{1118} ; ;$ p [gen3xFLAG-BiP $\left.{ }^{W T}\right]^{\text {AttP-89E11 }}$

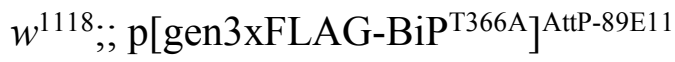

$w^{1118 ; ;}$ [ $\left[\text { gen3xFLAG-BiP }{ }^{\mathrm{T} 518 \mathrm{~A}}\right]^{\mathrm{AttP}-89 \mathrm{E} 11}$

$w^{1118} ;$ fic $^{30 \mathrm{C}} ; \mathrm{p}\left[\mathrm{gen} 3 \mathrm{xFLAG-BiP}{ }^{\mathrm{WT}}\right]^{\mathrm{AttP}-89 \mathrm{E} 11}$

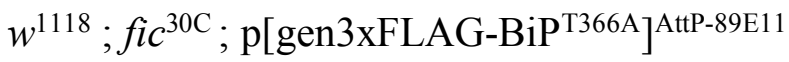

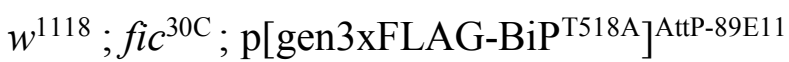

$B i P^{\mathrm{G} 0102} ; ; \mathrm{p}\left[\mathrm{gen} 3 \times \mathrm{xLAG}-\mathrm{BiP} \mathrm{P}^{\mathrm{WT}}\right]^{\mathrm{AtP}-89 \mathrm{E} 11}$

$B i P^{G 0102} ; ;$ p[gen3xFLAG-BiP $\left.{ }^{\mathrm{T} 366 \mathrm{~A}}\right]^{\mathrm{AtP} P-89 \mathrm{E} 11}$

$B i P^{\mathrm{G} 0102} ; \mathrm{p}\left[G M R\right.$-dsRNA $\left.{ }^{\text {white }}\right] ; \mathrm{p}\left[\mathrm{gen} 3 \mathrm{xFLAG-BiP^{ \textrm {WT } }}\right]^{89 \mathrm{E} 11}$

$B i P^{\mathrm{G} 0102} ; \mathrm{p}\left[G M R\right.$-dsRNA $\left.{ }^{\text {white }}\right] ; \mathrm{p}\left[\mathrm{Attb} \_ \text {gen3xFLAG-BiP }{ }^{\mathrm{T} 366 \mathrm{~A}}\right]^{89 \mathrm{E} 11}$

$w^{1118} ; \mathrm{p}\left[\right.$ tub-Atf $4^{5^{\prime} U T R}$-dsRed $] / \mathrm{p}\left[\right.$ GMR-dsRNA $\left.{ }^{\text {white }}\right]$

$w^{1118} ; f i c^{30 \mathrm{C}}, \mathrm{p}\left[t u b-A t f 4^{5^{\prime} U T R}\right.$-dsRed $] / \mathrm{fic}^{30 \mathrm{C}}, \mathrm{p}\left[G M R\right.$-dsRNA $\left.{ }^{\text {white }}\right]$

$w^{1118} ; \mathrm{p}\left[\right.$ GMR-dsRNA $\left.{ }^{\text {white }}\right] ; \mathrm{p}[D a-G a 14] / \mathrm{p}\left[\mathrm{UAS}^{\text {Scer-Xbp1-GFP.hg }]}\right.$

$w^{1118} ;$ fic $^{30 \mathrm{C}}, \mathrm{p}\left[\right.$ GMR-dsRNA $\left.{ }^{\text {white }}\right] ; \mathrm{p}[D a-\mathrm{Gal} 4] / \mathrm{p}\left[\mathrm{UAS}^{\text {Scer-Xbp1-GFP.hg }]}\right.$ 


\section{Generation of genomic BiP transgenes}

BiP cDNA sequence were subcloned into a pAttB vector and a $3 X-F L A G$ tag was

338 inserted after the N-terminal signal sequence. To create the T366A and T518A mutations,

339 gBlocks (IDT, Coralville, IA) for the mutant sequences were synthesized and subcloned into the

340 pAttB_genomic BiP vector via NEB HiFi Assembly Kit (NEB, Ipswich, MA). These constructs

341 were sequence-verified and injected into embryos (BestGene, Chino Hills, CA) for insertion at

342 the 89E11 landing site. Expression levels of FLAG-BiP transgenes were determined with

343 western blotting. In brief, fly heads were homogenized in lysis buffer (10\% SDS, 6M urea, and

$34450 \mathrm{mM}$ Tris-HCl, $\mathrm{pH} 6.8+10 \%$ DTT), sonicated for $5 \mathrm{~min}$, boiled for $2 \mathrm{~min}$, and centrifuged for

$34510 \mathrm{~min}$ at $10,000 \mathrm{~g}$ to remove debris. $10 \mu \mathrm{L}$ were separated by SDS-PAGE and transferred to

346 nitrocellulose membranes. Blots were probed with anti-BiP (1:8000, gift from Dr. Don Ryoo,

347 NYU, NY), anti-FLAG (1:2000 M2- F3165, Millipore Sigma, St. Louis, MO) and anti-Actin

348 (1:4000, JLA-20, DSHB, Iowa City, IA) and detected using IRdye-labeled antibodies and an

349 Odyssey scanner (LI-COR Biosciences, Lincoln, NE).

\section{Generation of GMR_dsRNA ${ }^{\text {white }}$ transgenes}

To make the eye-targeted dsRNA constructs against the white gene, the dsRNA sequence was obtained from a pAttb-UAS ${ }^{\text {S.cer-dsRNA }}{ }^{\text {white }}$ vector (a gift from Dr. Dean Smith, UT

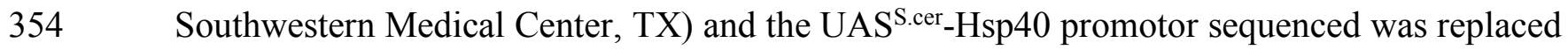
355 with a $5 \mathrm{X}-G M R$ promotor sequence, synthesized as a gBlock (IDT) and cloned with NEB HiFi 356 Assembly Kit (NEB). 


\section{Fly rearing conditions}

All flies were reared on standard molasses fly food, under room temperature conditions. For light treatments, flies were collected within one to two days of enclosing, and placed in $5 \mathrm{~cm}$ diameter vials containing normal food, with no more than 25 flies, and placed at either LD (lights ON 8am/lights OFF 8pm) or LL. ERGs, head dissections and behavior assays were performed between $1 \mathrm{pm}$ and $4 \mathrm{pm}$. The same intensity white LED light source was used for both conditions and flies were kept the same distance away from the light source, which amounted to approximately 500 lux. LD and LL treatments were done at $25^{\circ} \mathrm{C}$. For the UPR and Fic ${ }^{\mathrm{E} 247 \mathrm{G}}$ rough-eye interaction experiments, all flies were raised at $28^{\circ} \mathrm{C}$.

\section{Survival analysis of flies expressing genomic BiP construct}

$B i P^{\mathrm{G} 0102} / \mathrm{FM} 7 \mathrm{c}$ female virgin flies were crossed to males with either gen3xFLAG-BiP ${ }^{\mathrm{WT}}$, gen3xFLAG-BiP ${ }^{\mathrm{T} 366 \mathrm{~A}}$ or gen3xFLAG-BiP ${ }^{\mathrm{T} 518 \mathrm{~A}}$. The number of surviving non-FM7c male flies were scored by presence or lack of the Bar eye marker. Percent of expected was calculated from the actual number or recovered flies of the relevant genotypes compared the expected Mendelian number [\# observed flies/ \#expected flies]. Crosses were repeated three times ( $\mathrm{n}=3$ ). Total number of flies scored was at least 100 for each BiP variant.

\section{Survival analysis of flies expressing BiP variants in a $\mathrm{Da}$-Gal4, UAS-Fic ${ }^{\mathrm{E} 247 \mathrm{G}}$ background} C-terminally V5-His6-tagged UAS-Fic ${ }^{\mathrm{E} 247 \mathrm{G}}$ (Casey et al., 2017) was expressed via the ubiquitous $\mathrm{Da}$-Gal4 driver in $f i c^{30 \mathrm{C}} / \mathrm{CyO}$ heterozygous flies. These flies were crossed to $w^{;} ; f_{i c}{ }^{30 \mathrm{C}}$ (controls), $w$; fic $c^{30 \mathrm{C}}$; gen3xFLAGBiP ${ }^{\mathrm{WT}}$, w-; $f i c^{30 \mathrm{C}}$; gen3xFLAG ${ }^{\mathrm{T366A}}$, w-; $f i c^{30 \mathrm{C}}$; gen3xFLAG$\mathrm{BiP}^{\mathrm{T} 518 \mathrm{~A}}$, or $B i P^{\mathrm{G} 0102} ; \mathrm{fic}^{30 \mathrm{C}}$; gen3xFLAG ${ }^{\mathrm{T} 366 \mathrm{~A}}$. Offspring were scored and the number of adults 
381 homozygous for $\mathrm{fic}^{30 \mathrm{C}}$ with the $D a$-Gal4, UAS-Fic ${ }^{\mathrm{E} 247 \mathrm{G}}$ allele and were compared to the number

382 of $f i c^{30 \mathrm{C}}$ heterozygous sibling controls. Percent of expected was calculated from the actual

383 number or recovered flies of the relevant genotypes compared the expected Mendelian number

384 [\# observed flies/ \#expected flies]. Crosses were repeated three times $(\mathrm{n}=3)$. Total number of

385 flies scored was at least 100 for each $\mathrm{BiP}$ variant each repeat.

\section{Electroretinograms}

ERGs were recorded as previously described (Montell, 2012). Glass electrodes filled with $2 \mathrm{M} \mathrm{NaCl}$ were placed in the fly thorax and surface of the corneal lens (recording). A computercontrolled LED light source (MC1500; Schott, Mainz, Germany) was pulsed for 1s at 4s intervals. The resulting ERG traces were collected by an electrometer (IE-210; Warner

\section{Deep pseudopupil analysis}

Flies were anesthetized on $\mathrm{CO} 2$ and aligned with one eye facing up. Using a stereoscopic with Clampfit software (Molecular Devices). Flies were assayed in batches of eight to ten, and resulting quantifications are pooled from three independent biological repeats.

\section{2 biological replicas were performed $(n=3)$.}




\section{Light-startle behavior assay}

Assay was adapted from a previously described method (Ni et al., 2017). After 72 hours of LD or LL treatment, 16 flies per genotype were collected at the same time each morning and placed into individual Drosophila Assay Monitoring (DAM) chambers (TriKinetics Inc, Waltham, MA). The DAM monitors were placed into a dark incubator. Two hours later, a 500lux light was turned on by a timer for five minutes. Data was collected with DAMSystem3.0 and DAMFileScan11.0 (TriKinetics Inc). The resulting data was exported to Microsoft Excel and graphed in GraphPad Prism. Three replica experiments were averaged and plotted as Time (min) vs Average activity per $2 \min$. bin $(n=3)$. The change in response to light was calculated for each light pulse as [mean beam breaks for 10min. post-pulse] - [mean beam breaks for 10min. prepulse].

\section{Scanning electron microscopy}

SEMs of fly eyes were obtained as previously described (Wolff, 2011). Eyes were fixed in $2 \%$ paraformaldehyde, $2 \%$ glutaraldehyde, $0.2 \%$ Tween 20 , and $0.1 \mathrm{M}$ cacodylate buffer, $\mathrm{pH}$ 7.4, for 2 hours. Samples were washed four times with increasing ethanol (25-100\%) for 12 hours each followed by a series of hexamethyldisilazane washes (25-100\% in ethanol) for one hour each. Flies were air dried for 24 hours, mounted on SEM stubs, and the bodies were coated in fast-drying silver paint. Flies were sputter coated with a gold/pallidum mixture for $60 \mathrm{~s}$ and imaged at $900 \mathrm{X}$ magnification, with extra high tension set at $3.0 \mathrm{kV}$ on a scanning electron microscope (Sigma SEM; Carl Zeiss, Germany). Ten flies per genotype were mounted and three were imaged $(n=3)$. Blinding of the samples' identity to the user acquiring the images was performed. 


\section{Transmission electron microscopy}

TEMs of retina sections were performed as previously described (Jenny, 2011; Rahman et al., 2012). Briefly, $550 \mathrm{~nm}$ sections were cut and stained with toluidine blue to confirm

431 orientation and section depth. Blocks were subsequently thin-sectioned at $70 \mathrm{~nm}$ with a diamond 432 knife (Diatome, Hatfield, PA) on a Leica Ultracut 6 ultramicrotome (Leica Microsystems, 433 Wetzlar, Germany) and collected onto formvar-coated, glow-discharged copper grids, post434 stained with $2 \%$ aqueous uranyl acetate and lead citrate. Images were acquired on a Tecnai G2 435 spirit transmission electron microscope (FEI) equipped with a LaB6 source using a voltage of $436 \quad 120 \mathrm{kV}$. Blinding of the samples to the technicians performing the processing and the user 437 acquiring the images was performed. Two fly heads per genotype/condition and at least three 438 thin sections per sample were examined $(n=2)$. Samples were unmasked after the images were $439 \quad$ processed.

\section{Immunohistochemistry for $\mathrm{BiP}$ and UPR reporters}

Fly heads were dissected in HL3 hemolymph-like solution, fixed for four hours in ice443 cold $4 \%$ para-formaldehyde in filtered PBS, washed overnight in $25 \%$ (wt/vol) sucrose in 444 phosphate buffer ( $\mathrm{pH} 7.4)$, embedded in Optimal Cutting Temperature compound (EMS, 445 Hatfield, PA) frozen in dry ice and sectioned at $20-\mu \mathrm{m}$ thickness on a cryostat microtome (CM 446 1950, Leica Microsystems, Wetzlar, Germany). Sections were probed overnight with primary 447 antibodies against Drosophila BiP (1:2000, Gift from Don Ryoo(Ryoo et al.), GFP (1:1000, 448 A10262, ThermoFisher Scientific, Waltham, MA) or RFP (1:1000, 600-401-379, Rockland, 449 Limerick, PA). Secondary antibodies were labeled with Alexa488-conjugated Goat anti-Chicken 
450 (Molecular Probes, P/N\# A-11039), Alexa488-conjugated Goat anti-Guinea Pig (Molecular

451 Probes, P/N\# A-11073), or Alexa568-conjugated Goat anti-Rabbit (Molecular Probes, P/N\# A-

452 11011). Alexa 647-conjugated phallodin was also added to label Actin for identifying structures.

453 Images were captured with an oil-immersion $63 \times$ NA-1.4 lens on an inverted confocal

454 microscope (LSM710, Carl Zeiss). For each genotype and light rearing conditions,

455 immunohistochemistry experiments were performed in two biological replicas with new sets of

456 flies, using identical acquisition settings. Blinding of the samples to the user acquiring the

457 images was performed when appropriate.

Quantification of fluorescence staining

Fluorescence images were quantified using ImageJ (NIH) adapting previous methods

461 (Nandi et al., 2017). For each antibody, a threshold was determined, removing the lowest 10\% of

462 signal in LD control samples (to reduce variation from low level background signals). This same

463 threshold was applied, and a mask was created for every image in a batch of staining. Within a 1-

$464 \mu \mathrm{m}$ optical slice, the retina and lamina regions were selected manually using an Actin stain and

465 assigned as Regions of Interest. The integrated pixel intensity per unit area was measured within

466 this selected area, redirecting to the threshold mask. In each fly, four sequential optical slices

467 were quantified and averaged. For each genotype and treatment, four flies were quantified from

468 two independent biological replicas for a total of eight flies. Data was normalized to the wild-

469 type LD control for each replica. Outliers of greater than three standard deviations were omitted

$470 \quad$ from the analysis. 


\section{Yeast plasmids and strains}

473

Yeast genetic techniques were performed by standard procedures described previously. (Sherman et al., 1981). All strains were cultured in either rich (YPD: 1\% yeast extract, 2\% peptone, and $2 \%$ glucose) or complete synthetic minimal (CSM) media lacking appropriate amino acids with $2 \%$ glucose. Yeast were grown to log phase, serially diluted, and spotted onto agar plates to assay fitness and temperature sensitivity as previously described (Tran et al., 2007).

DNA fragments of KAR2 was generated by PCR amplification of the endogenous KAR2 gene using the primers 5'-GCATCCGCGGATACTCTCGTACCCTGCCGC-3' and 5'ATGCGAGCTCCGTAtAtACtCAgtAtAATC-3'. Plasmid pKAR2:LEU2 and pKAR2:URA3 were generated by subcloning genomic DNA fragments containing promoter and coding sequence of KAR2 into the SacI and SacII sites of pRS315 and pRS316, respectively. pKAR2T386A:LEU2 was generated by site directed mutagenesis of pKAR2:LEU2 using the primers 5'GGTTGGTGGTTCTGCTAGAATTCCAAAGGTCCAACAATTGTTAGAATCATACTTTGATGG-3' and 5'-ACСTTTGGAATTCTAGCAGAACCACCAACCAAAACGATATCATCAACATCCTTCTTTTCC-3'. pKAR2T538A:LEU2 was generated by site directed mutagenesis of pKAR2:LEU2 using the primers 5'-AGATAAGGGAGCTGGTAAATCCGAATCTATCACCATCACTAACG-3' and 5'GGATTTACCAGCTCCСTTATCTGTGGCAGACACCTTCAGAATACC-3’.

ACY008 yeast (mat A kar2::KAN his $3 \Delta 0$ leu2 $\Delta 0$ LYS met15 $\Delta 0$ ura3 $\Delta 0$ pKAR2:URA) were obtained by sporulation and dissection of KAR2 heterozygous null yeast (Mata/mat@, KAR2::KAN/KAR2 his3 $\Delta 0 /$ his $3 \Delta 0$ leu2 $\Delta 0 /$ leu2 $\Delta 0$ LYS/lys MET/met15 $\Delta 0$ ura3 $\Delta 0 /$ ura3 $\Delta 0$ ) (GE) transformed with pKAR2:URA. Standard plasmid shuffle techniques with 5-FOA(Zymo) 
were utilized to obtain ACY016 (mat A kar2::KAN his3 $\Delta 0$ leu2 $\Delta 0$ LYS met15 $\Delta 0$ ura3 $\Delta 0$ pKAR2:LEU2) ACY017(mat A kar2::KAN his3 $\Delta 0$ leu2 $\Delta 0$ LYS met15 $\Delta 0$ ura3 $\Delta 0$ pKAR2T386A:LEU2), and ACY020(mat A kar2::KAN his3 $\Delta 0$ leu2 $\Delta 0$ LYS met15 $\Delta 0$ ura3 $\Delta 0$ pKAR2T538A:LEU2)

\section{Statistics} determined using D'Agostino's \& Pearson's normality test. For the genetic analysis in Figure 1 and the ERG measurements in Supplemental Figures 2,3,5, and 6, significance was determined using one-way ANOVA, followed by Tukey's multiple comparisons tests. Statistical significance for non-parametric data, including the ERGs with light treatment quantifications in Figure 2, was determined by Kruskal-Wallis tests followed by multiple comparisons testing with Dunn's correction. For the image quantification data in Figure 4, significance was determined by twoway ANOVA followed by multiple comparisons with Benjamini-Krieger-Yekutieli's False Discovery Rate correction. All tests were two-sided with no experimental matching. RStudio (version 1.1.442, 2018, RStudio, Inc.) was used for Fisher's Exact Tests for the eye interaction screen, with Bonferroni’s multiple comparison method to determine significance. Standard R functions were used, no custom scripts were developed. Tests were two-sided. When possible, blinding of sample identities was performed for image acquisition and fluorescence intensity quantification. Sample sizes for ERG assays, EM experiments, fluorescence quantifications and fly genetic analysis were determined based from previous experience (Nandi et al., 2017; Rahman et al., 2012; Stenesen et al., 2015). 
bioRxiv preprint doi: https://doi.org/10.1101/348797; this version posted June 15, 2018. The copyright holder for this preprint (which was not certified by peer review) is the author/funder, who has granted bioRxiv a license to display the preprint in perpetuity. It is made available under aCC-BY 4.0 International license.

\section{$517 \quad$ Competing Interests:}

518 The authors declare no competing interests. 


\section{References:}

Akbar, M. A., Ray, S., \& Krämer, H. (2009). The SM protein Car/Vps33A regulates SNAREmediated trafficking to lysosomes and lysosome-related organelles. Mol Biol Cell, 20(6), 1705-1714. doi:10.1091/mbc.E08-03-0282

Amin-Wetzel, N., Saunders, R. A., Kamphuis, M. J., Rato, C., Preissler, S., Harding, H. P., \& Ron, D. (2017). A J-Protein Co-chaperone Recruits BiP to Monomerize IRE1 and Repress the Unfolded Protein Response. Cell, 171(7), 1625-1637 e1613. doi:10.1016/j.cell.2017.10.040

Bertolotti, A., Zhang, Y., Hendershot, L. M., Harding, H. P., \& Ron, D. (2000). Dynamic interaction of BiP and ER stress transducers in the unfolded-protein response. Nat Cell Biol, 2(6), 326-332. doi:10.1038/35014014

Borycz, J., Borycz, J. A., Loubani, M., \& Meinertzhagen, I. A. (2002). tan and ebony genes regulate a novel pathway for transmitter metabolism at fly photoreceptor terminals. $J$ Neurosci, 22(24), 10549-10557.

Brown, M. S., Segal, A., \& Stadtman, E. R. (1971). Modulation of glutamine synthetase adenylylation and deadenylylation is mediated by metabolic transformation of the P II regulatory protein. Proc Natl Acad Sci US A, 68(12), 2949-2953.

Carrara, M., Prischi, F., Nowak, P. R., Kopp, M. C., \& Ali, M. M. (2015). Noncanonical binding of BiP ATPase domain to Ire 1 and Perk is dissociated by unfolded protein $\mathrm{CH} 1$ to initiate ER stress signaling. Elife, 4. doi:10.7554/eLife.03522

Casey, A. K., Moehlman, A. T., Zhang, J., Servage, K. A., Krämer, H., \& Orth, K. (2017). Ficmediated deAMPylation is not dependent on homodimerization and rescues toxic AMPylation in flies. J Biol Chem, 292(51), 21193-21204. doi:10.1074/jbc.M117.799296

Casey, A. K., \& Orth, K. (2018). Enzymes Involved in AMPylation and deAMPylation. Chem Rev, 118(3), 1199-1215. doi:10.1021/acs.chemrev.7b00145

Colley, N. J., Cassill, J. A., Baker, E. K., \& Zuker, C. S. (1995). Defective intracellular transport is the molecular basis of rhodopsin-dependent dominant retinal degeneration. Proc Natl Acad Sci US A, 92(7).

Damulewicz, M., Mazzotta, G. M., Sartori, E., Rosato, E., Costa, R., \& Pyza, E. M. (2017). Cryptochrome Is a Regulator of Synaptic Plasticity in the Visual System of Drosophila melanogaster. Front Mol Neurosci, 10, 165. doi:10.3389/fnmol.2017.00165

Dietzl, G., Chen, D., Schnorrer, F., Su, K. C., Barinova, Y., Fellner, M., . . . Dickson, B. J. (2007). A genome-wide transgenic RNAi library for conditional gene inactivation in Drosophila. Nature, 448(7150), 151-156. doi:10.1038/nature05954

Dolph, P. J., Ranganathan, R., Colley, N. J., Hardy, R. W., Socolich, M., \& Zuker, C. S. (1993). Arrestin function in inactivation of $\mathrm{G}$ protein-coupled receptor rhodopsin in vivo. Science, 260(5116), 1910-1916.

Franceschini, N., \& Kirschfeld, K. (1971). [Pseudopupil phenomena in the compound eye of drosophila]. Kybernetik, 9(5), 159-182.

Gardner, B. M., Pincus, D., Gotthardt, K., Gallagher, C. M., \& Walter, P. (2013). Endoplasmic reticulum stress sensing in the unfolded protein response. Cold Spring Harb Perspect Biol, 5(3), a013169. doi:10.1101/cshperspect.a013169

Gaut, J. R., \& Hendershot, L. M. (1993). Mutations within the nucleotide binding site of immunoglobulin-binding protein inhibit ATPase activity and interfere with release of immunoglobulin heavy chain. J Biol Chem, 268(10), 7248-7255. 
Ham, H., Woolery, A. R., Tracy, C., Stenesen, D., Krämer, H., \& Orth, K. (2014). Unfolded protein response-regulated Drosophila Fic (dFic) protein reversibly AMPylates BiP chaperone during endoplasmic reticulum homeostasis. J Biol Chem, 289(52), 3605936069. doi:10.1074/jbc.M114.612515

Harms, A., Stanger, F. V., \& Dehio, C. (2016). Biological Diversity and Molecular Plasticity of FIC Domain Proteins. Annu Rev Microbiol, 70, 341-360. doi:10.1146/annurev-micro102215-095245

Hendershot, L. M., Ting, J., \& Lee, A. S. (1988). Identity of the immunoglobulin heavy-chainbinding protein with the 78,000-dalton glucose-regulated protein and the role of posttranslational modifications in its binding function. Mol Cell Biol, 8(10), 4250-4256.

Jenny, A. (2011). Preparation of adult Drosophila eyes for thin sectioning and microscopic analysis. J Vis $\operatorname{Exp}(54)$. doi:10.3791/2959

Johnson, K., Grawe, F., Grzeschik, N., \& Knust, E. (2002). Drosophila crumbs is required to inhibit light-induced photoreceptor degeneration. Curr Biol, 12(19), 1675-1680.

Kang, K., Ryoo, H. D., Park, J. E., Yoon, J. H., \& Kang, M. J. (2015). A Drosophila Reporter for the Translational Activation of ATF4 Marks Stressed Cells during Development. PLoS One, 10(5), e0126795. doi:10.1371/journal.pone.0126795

Kingdon, H. S., Shapiro, B. M., \& Stadtman, E. R. (1967). Regulation of glutamine synthetase. 8. ATP: glutamine synthetase adenylyltransferase, an enzyme that catalyzes alterations in the regulatory properties of glutamine synthetase. Proc Natl Acad Sci U S A, 58(4), 17031710.

Kiselev, A., Socolich, M., Vinos, J., Hardy, R. W., Zuker, C. S., \& Ranganathan, R. (2000). A molecular pathway for light-dependent photoreceptor apoptosis in Drosophila. Neuron, 28(1), 139-152.

Kozutsumi, Y., Segal, M., Normington, K., Gething, M. J., \& Sambrook, J. (1988). The presence of malfolded proteins in the endoplasmic reticulum signals the induction of glucoseregulated proteins. Nature, 332(6163), 462-464. doi:10.1038/332462a0

Lee, S. J., \& Montell, C. (2004). Suppression of constant-light-induced blindness but not retinal degeneration by inhibition of the rhodopsin degradation pathway. Curr Biol, 14(23), 2076-2085. doi:10.1016/j.cub.2004.11.054

Lee, S. J., Xu, H., Kang, L. W., Amzel, L. M., \& Montell, C. (2003). Light adaptation through phosphoinositide-regulated translocation of Drosophila visual arrestin. Neuron, 39(1), 121-132.

Leonard, D. S., Bowman, V. D., Ready, D. F., \& Pak, W. L. (1992). Degeneration of photoreceptors in rhodopsin mutants of Drosophila. J Neurobiol, 23(6), 605-626. doi:10.1002/neu.480230602

Ma, Y., \& Hendershot, L. M. (2003). Delineation of a negative feedback regulatory loop that controls protein translation during endoplasmic reticulum stress. J Biol Chem, 278(37), 34864-34873. doi:10.1074/jbc.M301107200

Meunier, L., Usherwood, Y. K., Chung, K. T., \& Hendershot, L. M. (2002). A subset of chaperones and folding enzymes form multiprotein complexes in endoplasmic reticulum to bind nascent proteins. Mol Biol Cell, 13(12), 4456-4469. doi:10.1091/mbc.E02-050311

Montell, C. (2012). Drosophila visual transduction. Trends Neurosci, 35(6), 356-363. doi:10.1016/j.tins.2012.03.004 
610

Nandi, N., Tyra, L. K., Stenesen, D., \& Krämer, H. (2017). Stress-induced Cdk5 activity enhances cytoprotective basal autophagy in Drosophila melanogaster by phosphorylating acinus at serine(437). Elife, 6. doi:10.7554/eLife.30760

Ni, J. D., Baik, L. S., Holmes, T. C., \& Montell, C. (2017). A rhodopsin in the brain functions in circadian photoentrainment in Drosophila. Nature, 545(7654), 340-344. doi: $10.1038 /$ nature22325

Preissler, S., Rato, C., Chen, R., Antrobus, R., Ding, S., Fearnley, I. M., \& Ron, D. (2015). AMPylation matches BiP activity to client protein load in the endoplasmic reticulum. Elife, 4, e12621. doi:10.7554/eLife.12621

Preissler, S., Rato, C., Perera, L., Saudek, V., \& Ron, D. (2017). FICD acts bifunctionally to AMPylate and de-AMPylate the endoplasmic reticulum chaperone BiP. Nat Struct Mol Biol, 24(1), 23-29. doi:10.1038/nsmb.3337

Preissler, S., Rohland, L., Yan, Y., Chen, R., Read, R. J., \& Ron, D. (2017). AMPylation targets the rate-limiting step of BiP's ATPase cycle for its functional inactivation. Elife, 6 . doi:10.7554/eLife.29428

Rahman, M., Ham, H., Liu, X., Sugiura, Y., Orth, K., \& Kramer, H. (2012). Visual neurotransmission in Drosophila requires expression of Fic in glial capitate projections. Nat Neurosci, 15(6), 871-875. doi:10.1038/nn.3102

Rosenbaum, E. E., Hardie, R. C., \& Colley, N. J. (2006). Calnexin is essential for rhodopsin maturation, $\mathrm{Ca} 2+$ regulation, and photoreceptor cell survival. Neuron, 49(2), 229-241. doi:10.1016/j.neuron.2005.12.011

Ryoo, H. D., Domingos, P. M., Kang, M. J., \& Steller, H. (2007). Unfolded protein response in a Drosophila model for retinal degeneration. EMBO J, 26(1), 242-252. doi:10.1038/sj.emboj.7601477

Scott, K., Becker, A., Sun, Y., Hardy, R., \& Zuker, C. (1995). Gq alpha protein function in vivo: genetic dissection of its role in photoreceptor cell physiology. Neuron, 15(4), 919-927.

Shen, J., Snapp, E. L., Lippincott-Schwartz, J., \& Prywes, R. (2005). Stable binding of ATF6 to $\mathrm{BiP}$ in the endoplasmic reticulum stress response. Mol Cell Biol, 25(3), 921-932. doi:10.1128/MCB.25.3.921-932.2005

Sherman, F., Fink, G. R., Hicks, J. B., \& Laboratory, C. S. H. (1981). Methods in yeast genetics: Cold Spring Harbor Laboratory.

Smith, D. P., Ranganathan, R., Hardy, R. W., Marx, J., Tsuchida, T., \& Zuker, C. S. (1991). Photoreceptor deactivation and retinal degeneration mediated by a photoreceptor-specific protein kinase C. Science, 254(5037), 1478-1484.

Sone, M., Zeng, X., Larese, J., \& Ryoo, H. D. (2013). A modified UPR stress sensing system reveals a novel tissue distribution of IRE1/XBP1 activity during normal Drosophila development. Cell Stress Chaperones, 18(3), 307-319. doi:10.1007/s12192-012-0383-x

Soukup, S. F., Pocha, S. M., Yuan, M., \& Knust, E. (2013). DLin-7 is required in postsynaptic lamina neurons to prevent light-induced photoreceptor degeneration in Drosophila. Curr Biol, 23(14), 1349-1354. doi:10.1016/j.cub.2013.05.060

Stenesen, D., Moehlman, A. T., \& Krämer, H. (2015). The carcinine transporter CarT is required in Drosophila photoreceptor neurons to sustain histamine recycling. Elife, 4, e10972. doi:10.7554/eLife.10972

Sugie, A., Hakeda-Suzuki, S., Suzuki, E., Silies, M., Shimozono, M., Mohl, C., . . Tavosanis, G. (2015). Molecular Remodeling of the Presynaptic Active Zone of Drosophila 
Photoreceptors via Activity-Dependent Feedback. Neuron, 86(3), 711-725. doi:10.1016/j.neuron.2015.03.046

Tran, E. J., Zhou, Y., Corbett, A. H., \& Wente, S. R. (2007). The DEAD-box protein Dbp5 controls mRNA export by triggering specific RNA:protein remodeling events. Mol Cell, 28(5), 850-859. doi:10.1016/j.molcel.2007.09.019

Truttmann, M. C., Cruz, V. E., Guo, X., Engert, C., Schwartz, T. U., \& Ploegh, H. L. (2016). The Caenorhabditis elegans Protein FIC-1 Is an AMPylase That Covalently Modifies HeatShock 70 Family Proteins, Translation Elongation Factors and Histones. PLoS Genet, 12(5), e1006023. doi:10.1371/journal.pgen.1006023

Truttmann, M. C., Zheng, X., Hanke, L., Damon, J. R., Grootveld, M., Krakowiak, J., .. . Ploegh, H. L. (2017). Unrestrained AMPylation targets cytosolic chaperones and activates the heat shock response. Proc Natl Acad Sci U S A, 114(2), E152-E160. doi:10.1073/pnas.1619234114

Venken, K. J., He, Y., Hoskins, R. A., \& Bellen, H. J. (2006). P[acman]: a BAC transgenic platform for targeted insertion of large DNA fragments in D. melanogaster. Science, 314(5806), 1747-1751. doi:10.1126/science.1134426

Wieteska, L., Shahidi, S., \& Zhuravleva, A. (2017). Allosteric fine-tuning of the conformational equilibrium poises the chaperone BiP for post-translational regulation. Elife, 6 . doi:10.7554/eLife. 29430

Wolff, T. (2011). Preparation of Drosophila eye specimens for scanning electron microscopy. Cold Spring Harb Protoc, 2011(11), 1383-1385. doi:10.1101/pdb.prot066506

Woolery, A. R., Yu, X., LaBaer, J., \& Orth, K. (2014). AMPylation of Rho GTPases subverts multiple host signaling processes. J Biol Chem, 289(47), 32977-32988. doi:10.1074/jbc.M114.601310

\section{Acknowledgments:}

We thank Drs. Eric Olson and Joe Takahashi and the members of the Kramer and Orth labs for discussion and technical assistance. We thank the Bloomington Stock Center (NIH P40OD018537) and the Vienna Drosophila Resource Center (VDRC, www.vdrc.at) for flies and the Molecular and Cellular Imaging Facility at the University of Texas Southwestern Medical center for help with electron microscopy (NIH S10 OD020103-01).

Funding: K.O. is a Burroughs Welcome Investigator in Pathogenesis of Infectious Disease, a Beckman Young Investigator, and a W. W. Caruth, Jr., Biomedical Scholar and has an Earl A. Forsythe Chair in Biomedical Science. 
$694 \quad$ Author contributions:

695 A.T.M, A.C. K.O and H.K. conceived, designed, and analyzed experiments and wrote the

696 manuscript. All experiments except yeast experiments were performed by A.T.M. A.C. and K.S.

697 performed mass spec experiments.

698

699 Data and materials availability: Fly stocks are available upon request. All data are contained $700 \quad$ in the source files.

701

702 Correspondence to: Helmut.Kramer@UTSouthwestern.edu and

$703 \quad$ Kim.Orth@utsouthwestern.edu. 
Figures
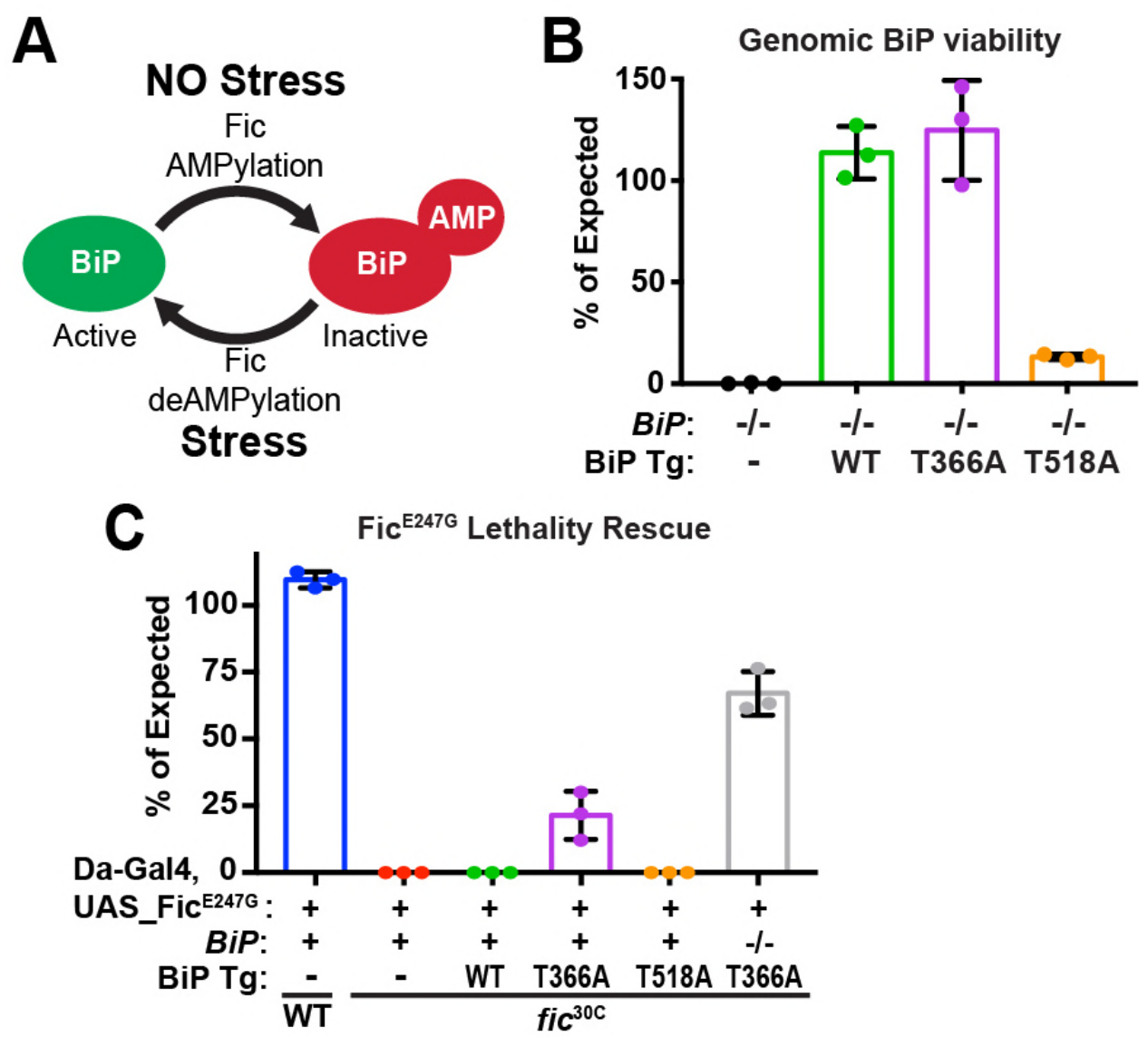

Figure 1. BiP is a target of Fic AMPylation and deAMPylation in vivo. (A) BiP AMPylation during times of low ER stress reserves a portion of the chaperone to allow for a rapid, deAMPylation-driven, response to high ER stress (Casey et al., 2017; Preissler, Rato, et al., 2017). (B) Bar graphs show the percentage of null mutant $B i P^{\mathrm{G} 0102} / y$ males rescued by the indicated genomic $\mathrm{BiP}^{\mathrm{WT}}$, $\mathrm{BiP}^{\mathrm{T} 366 \mathrm{~A}}$ or $\mathrm{BiP}^{\mathrm{T} 518 \mathrm{~A}}$ genomic transgene $(\mathrm{Tg})$ relative to sibling controls. $\mathrm{N}=3$ biological replicas. At least 50 flies scored for each replica. Bar graphs show means +/- Standard Deviation (SD). (C) Bar graphs show the percentage of viable flies of the indicated wild type or $\mathrm{fic}^{30 \mathrm{C}}$ genotypes expressing the overactive $\mathrm{Fic}^{\mathrm{E} 247 \mathrm{G}}$ under the ubiquitous Da-Gal4 driver relative to sibling controls. Among the indicated genomic BiP transgenes, only $\mathrm{BiP}^{\mathrm{T} 366 \mathrm{~A}}$ provides partial rescue of lethality in the $\mathrm{Bi}^{+/+}$background and near complete rescue in a $B i P^{\mathrm{G} 0102}$ null background. $\mathrm{N}=3$ biological replicas. At least 100 total flies scored for each replica. Bar graphs show means $+/-$ SD. 
Genotype: fic $^{30 \mathrm{C}} /+$; LongGMR-Gal4/UAS-RNAi-:

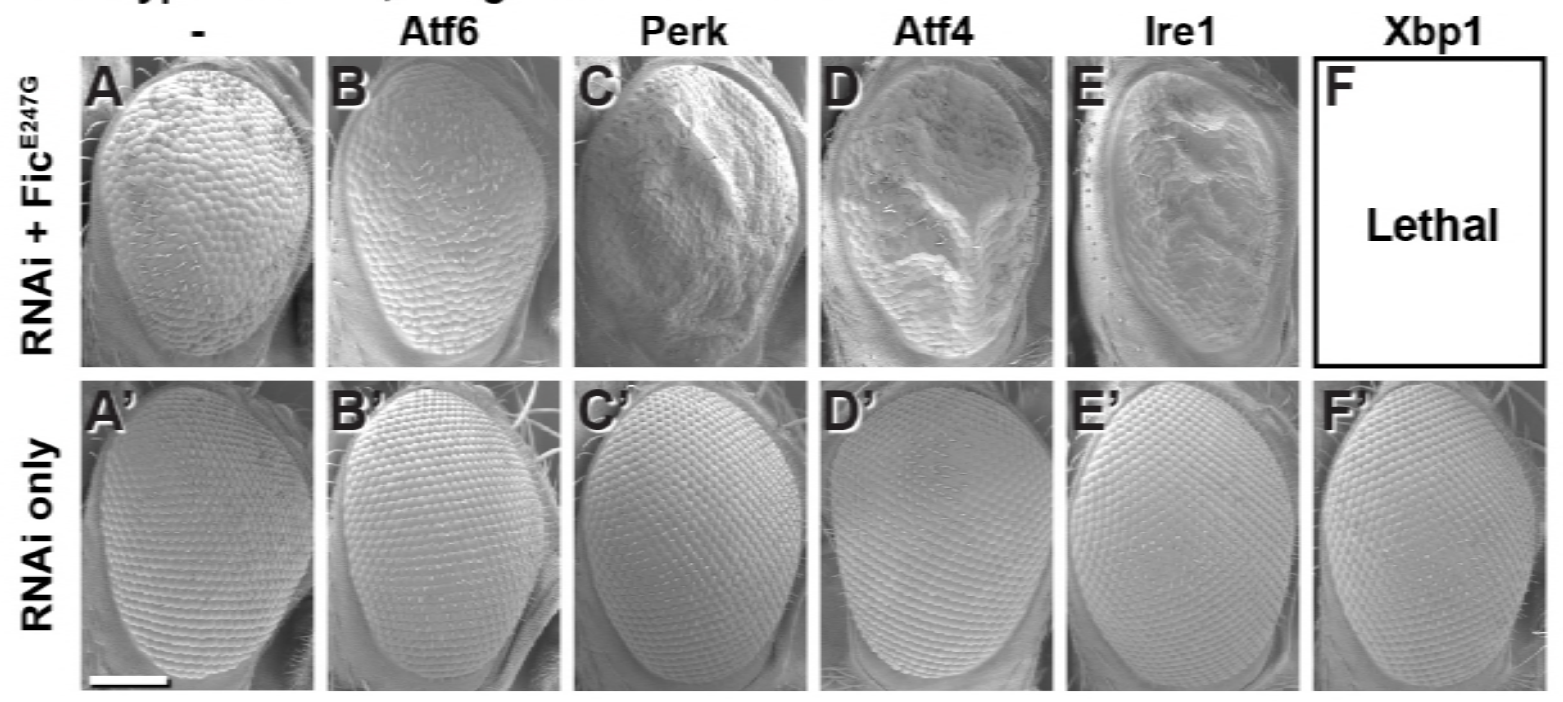

Figure 2. Genetic interactions between Fic and UPR genes. Representative SEM images of heterozygote mutant $\mathrm{fic}^{30 \mathrm{C} /+}$ eyes expressing the indicated UAS-RNAi transgenes with (A-F) or without (A'-F') UAS-Fic ${ }^{\mathrm{E} 247 \mathrm{G}}$ under longGMR-Gal4 control. See Supplemental Table 1 for 723 quantification. Scale bar: $100 \mu \mathrm{M}$. 


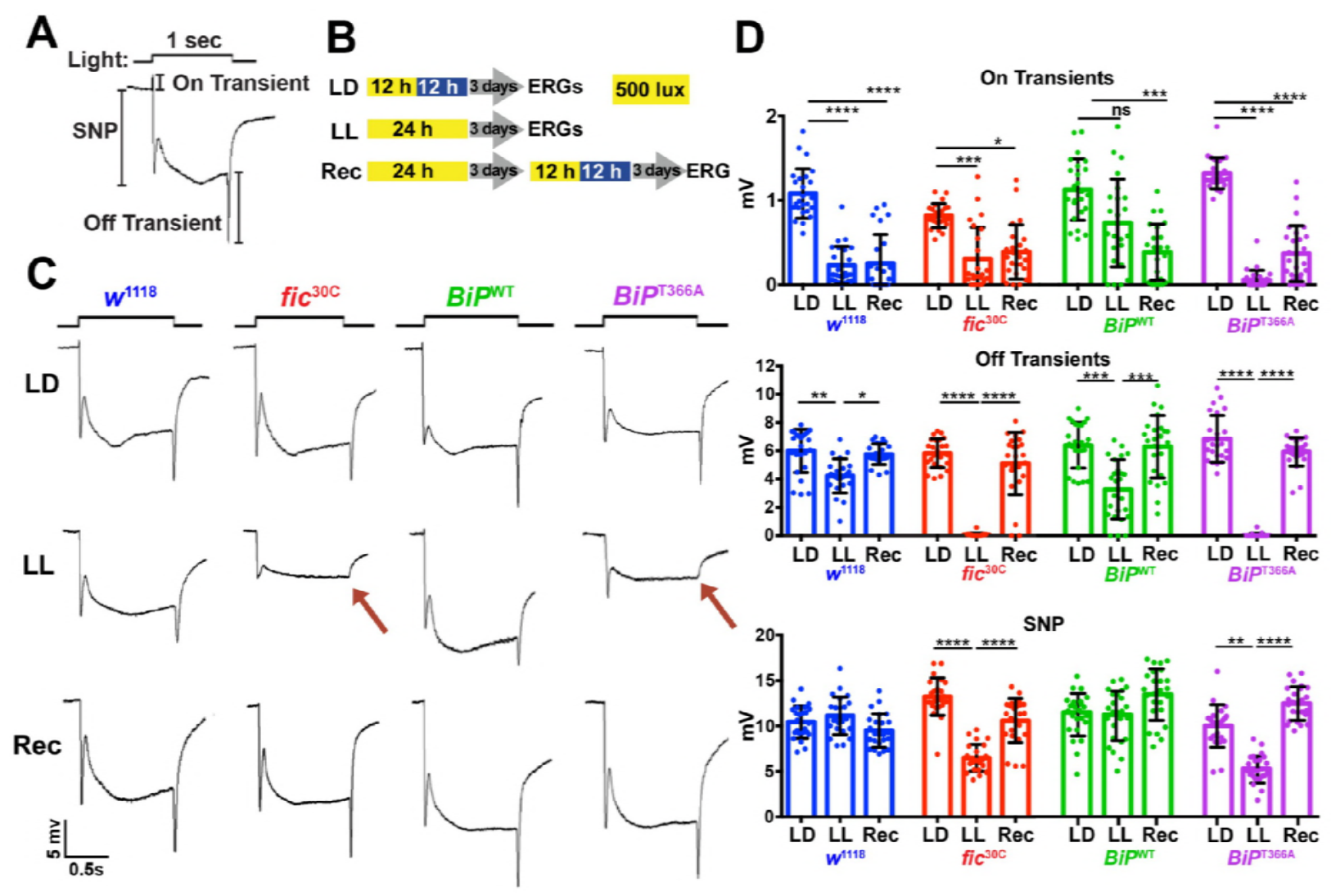

A representative ERG trace in response to a 1-sec light pulse displaying the sustained negative potential (SNP), representing the depolarization within photoreceptor neurons, and the ON \& OFF transients, reflecting post-synaptic activity of lamina neurons. (B) Representation of the different light treatments of flies before ERG recordings: 3 days of $12 \mathrm{hr}$ light (500 lux) and 12hr dark (LD), 3 days of continuous light (LL) or 3 days of continuous light followed by 3 days of LD (Rec). 1-sec light pulses were performed at $4 \mathrm{sec}$ intervals. (C) Representative traces from $w^{1118}, f i c^{30 \mathrm{C}}, B i P^{\mathrm{WT}}$ and $B i P^{\mathrm{T} 366 \mathrm{~A}}$ flies. Under LL, $f i c^{30 \mathrm{C}}$ and $B i P^{\mathrm{T} 366 \mathrm{~A}}$ mutants lose ON and OFF transients (red arrows) and have reduced SNPs. The changes are reversed after 3-days of recovery (Rec). (D) Quantification of key components of ERGs shown in panel C. Bar graphs show means +/- SD. ****, $\mathrm{p}<0.0001 ; * * *, \mathrm{p}<0.001 ; * *, \mathrm{p}<0.01 ; *, \mathrm{p}<0.05 ; \mathrm{n}=24$ flies for each genotype/condition, pooled from three independent biological replicas. 

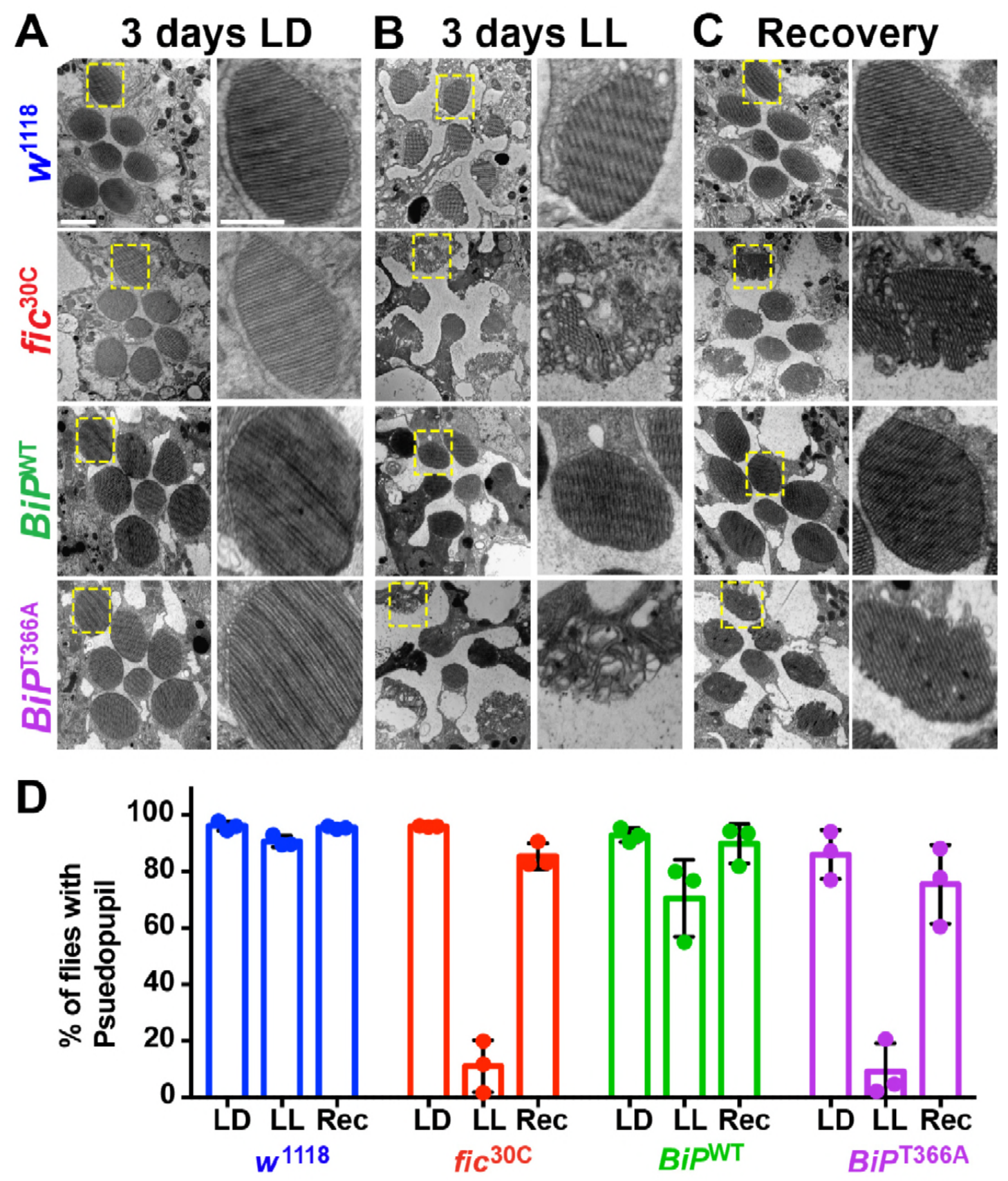

Figure 4. AMPylation of $\mathrm{BiP}$ is required for maintaining structural plasticity in the retina.

(A-C) Representative TEM images of retina thin sections from the indicated genotypes with either standard LD (A), the stress-inducing LL (B) or recovery treatment (C). Scale bars: $1 \mu \mathrm{M}$.

741 Yellow boxes indicate rhabdomeres shown in high magnification images. High magnification scale bars: $0.5 \mu \mathrm{M}$. (D) Percentages of flies with intact deep pseudopupil following LD, LL and Rec. $\mathrm{N}=3$ independent biological replicas with approximately 50 flies scored per genotype per replica. Bar graphs show means $+/-\mathrm{SD}$. 
A

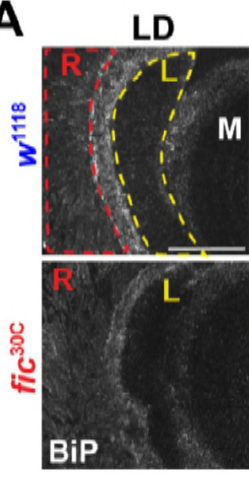

C
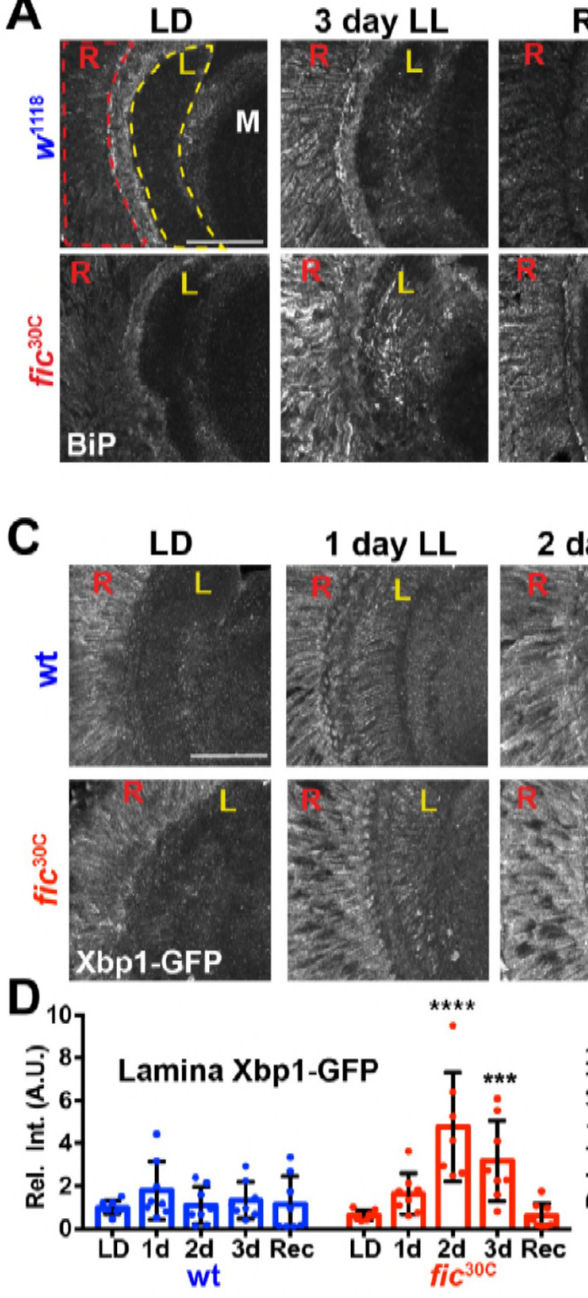
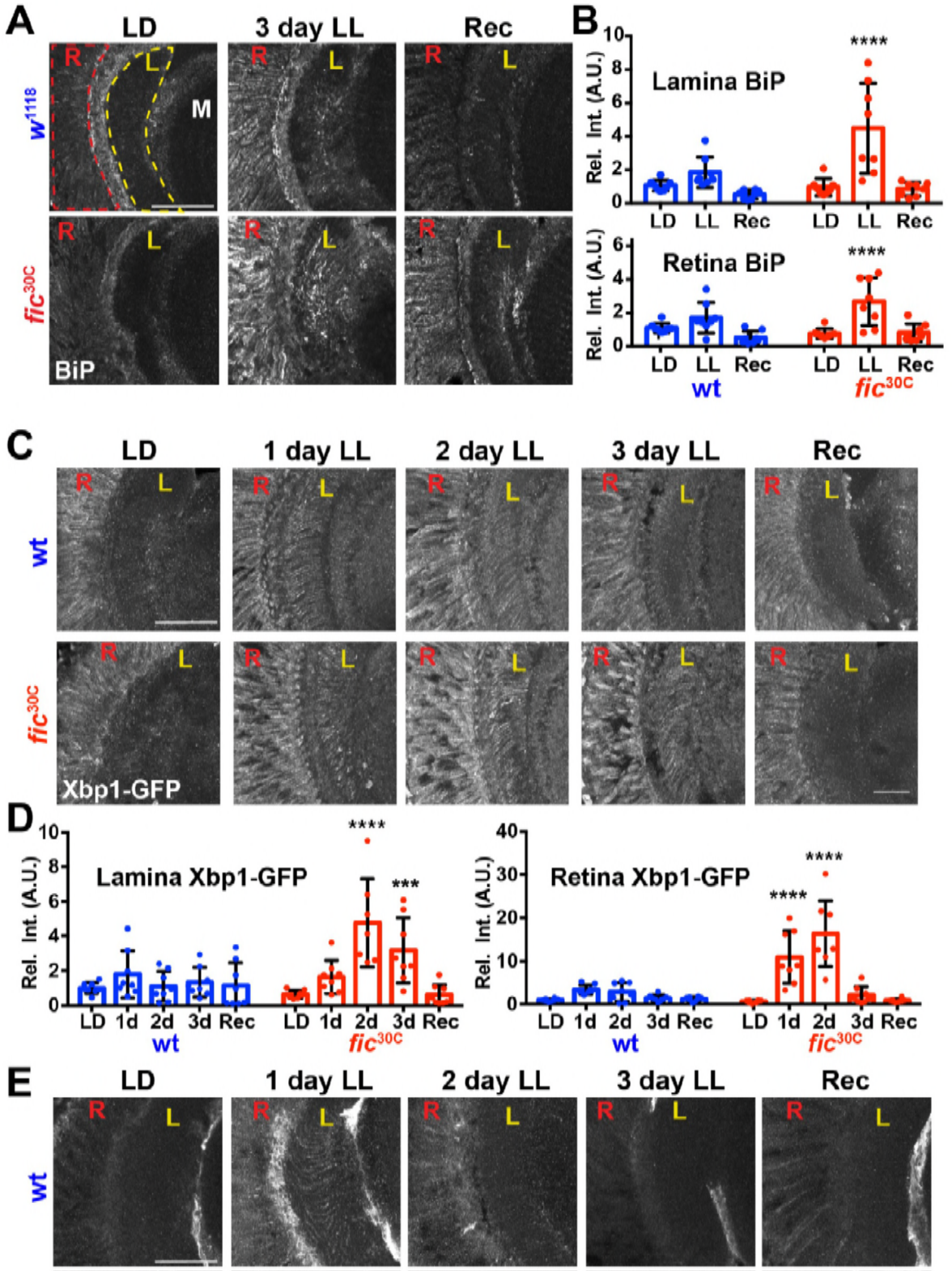

2 day LL

3 day LL
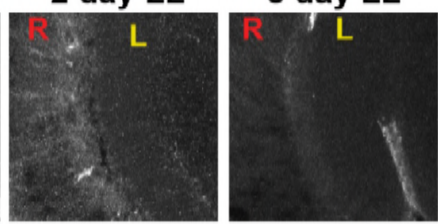

Rec
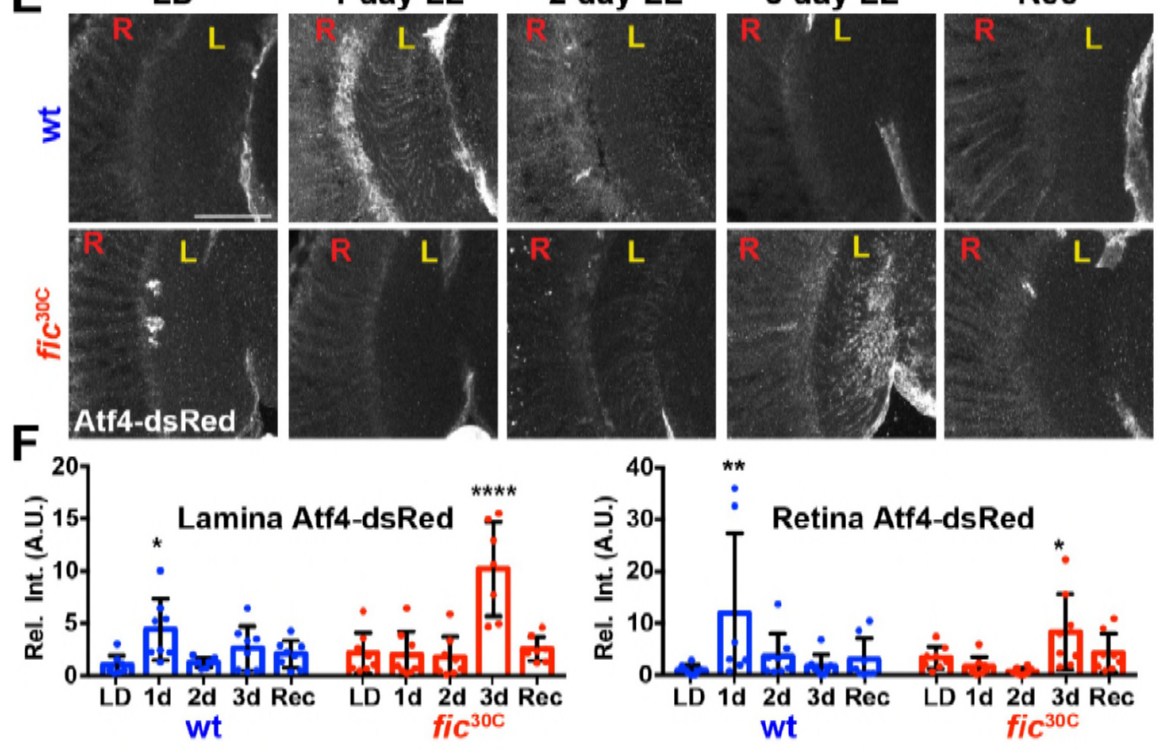

Figure 5. BiP AMPylation regulates ER homeostasis during prolonged light stimulation. 
following 3 days LD, LL or Recovery treatments. (B) Quantification of BiP fluorescence intensity, normalized to wild-type LD controls, in the lamina neuropil and retina from 2 independent experiments. (C) Representative images of a Xbp1-GFP splicing reporter in either a Fic wild-type or the null fic ${ }^{30 \mathrm{C}}$ background following LD, 1-day LL, 2-day LL, 3-day LL, and Recovery conditions. (D) Quantification of GFP fluorescence intensity, normalized to wild-type

753 LD controls, in the lamina neuropil and retina from 2 independent experiments. (E)

754 Representative images of an Atf4-dsRed reporter in either a wild-type or fic ${ }^{30 \mathrm{C}}$ background 755 following LD, 1-day LL, 2-day LL, 3-day LL, and Recovery conditions. (F) Quantification of Atf4-dsRed intensity, normalized to wild-type LD controls, in the lamina neuropil and retina from 2 independent experiments. For all experiments, $n=8$ flies per genotype/condition, with exceptions of outliers falling 3 standard deviations outside the mean. Bar graphs show means $+/-$ SD. For all experiments, significance is indicated for treatment compared to the LD condition for the corresponding genotype. ****, $\mathrm{p}<0.0001$; ***, $\mathrm{p}<0.001 ; * *, \mathrm{p}<0.01 ; *, \mathrm{p}<0.05$. All scale $761 \quad$ bars: $50 \mu \mathrm{M}$. 
A

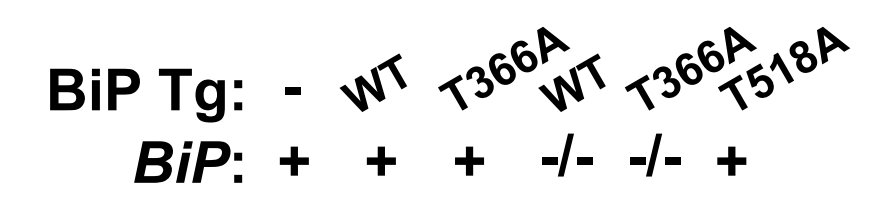

$70 \mathrm{kDa}-=====\mathrm{BiP}$

$70 \mathrm{kDa}-\cdots-\cdots-$ FLAG

$40 \mathrm{kDa}-ニ-ー \ldots$ Actin

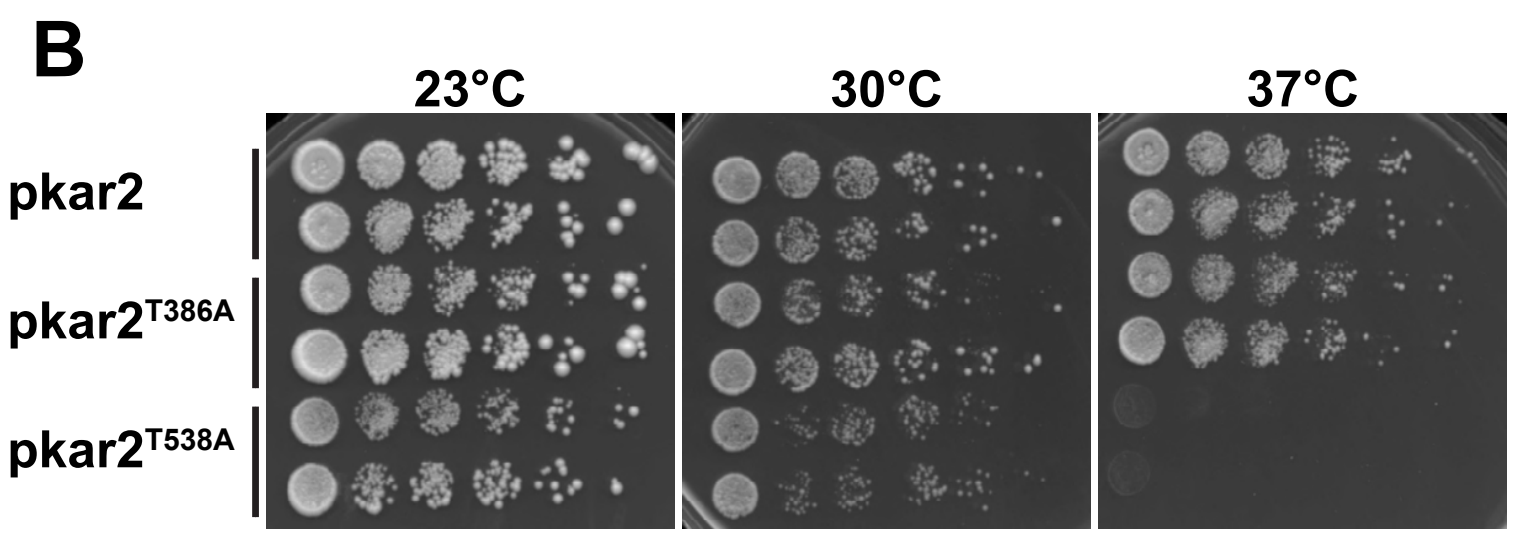

763 Figure 1- figure supplement 1. Expression of genomic BiP transgenes

764 (A) Western blots for FLAG-tagged BiP transgenes and total BiP in whole head lysates in BiP 765 wild type or homozygous mutant background as indicated. Actin (JLA-20) served as a loading 766 control. (B) Kar2 $2^{\mathrm{T} 538 \mathrm{~A}}$ mutants have temperature-sensitive growth defects. Yeast strains $k a r 2 \Delta$ $767+\mathrm{p} \operatorname{Kar} 2, \operatorname{kar} 2 \Delta+$ pkar2 $2^{\mathrm{T} 386 \mathrm{~A}}$, and $\operatorname{kar} 2 \Delta+\operatorname{pkar} 2^{\mathrm{T} 538 \mathrm{~A}}$ were grown at $25^{\circ} \mathrm{C}$ and five-fold serially 768 diluted onto plates of rich media incubated at the indicated temperatures. 

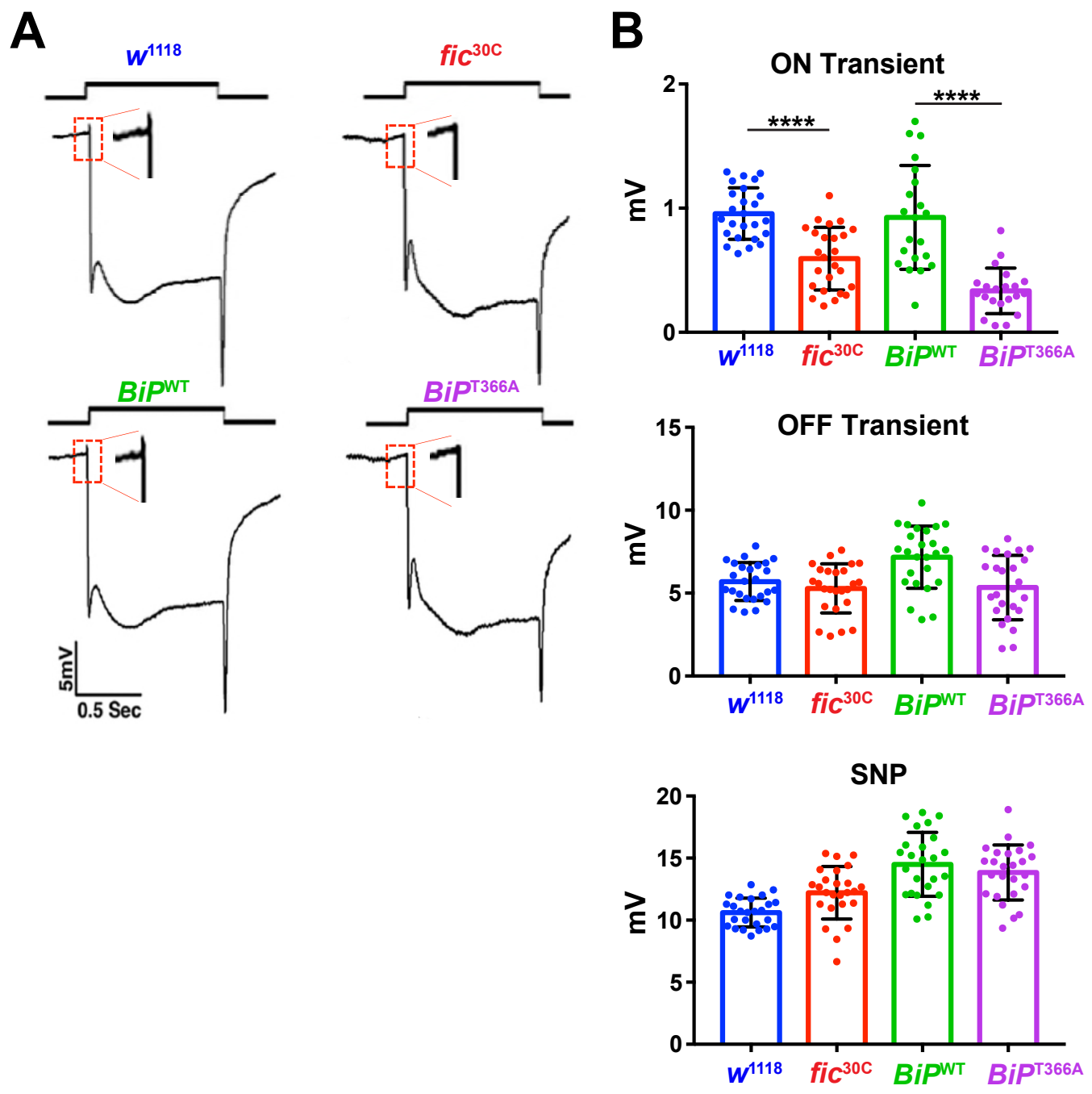

Figure 1- figure supplement 2. AMPylation-resistant $B P^{\mathrm{T} 366 \mathrm{~A}}$ phenocopies fic.

(A) ERGs of $f i c^{30 \mathrm{C}}$ flies show reduced ON transients (arrows). Flies homozygous for a lethal $B i P^{\mathrm{G} 0102}$ allele rescued by $\mathrm{BiP} \mathrm{PT}^{\mathrm{WT}}$ transgene have normal vision but flies rescued with the mutant

$773 \quad$ BiP $^{\mathrm{T} 366 \mathrm{~A}}$ transgene display reduced ON transients. (B) Quantification of ERG traces. Bar graphs show means +/- SD. ****, $\mathrm{p}<0.0001 ; * * *, \mathrm{p}<0.001 ; *, \mathrm{p}<0.05 ; \mathrm{n}=24$ flies per genotype and condition. 
A
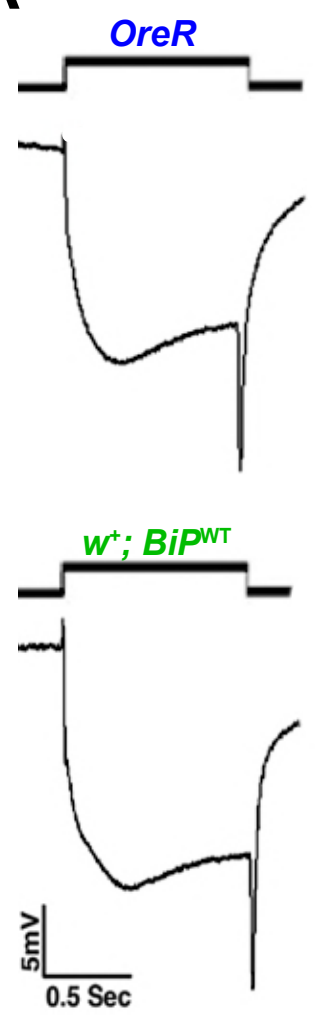

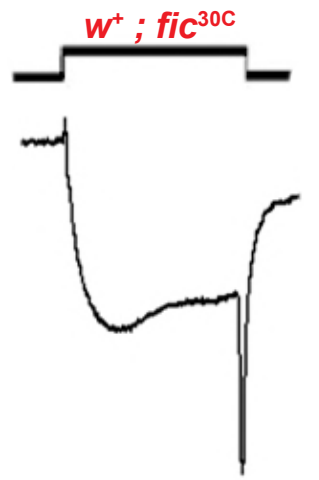

B
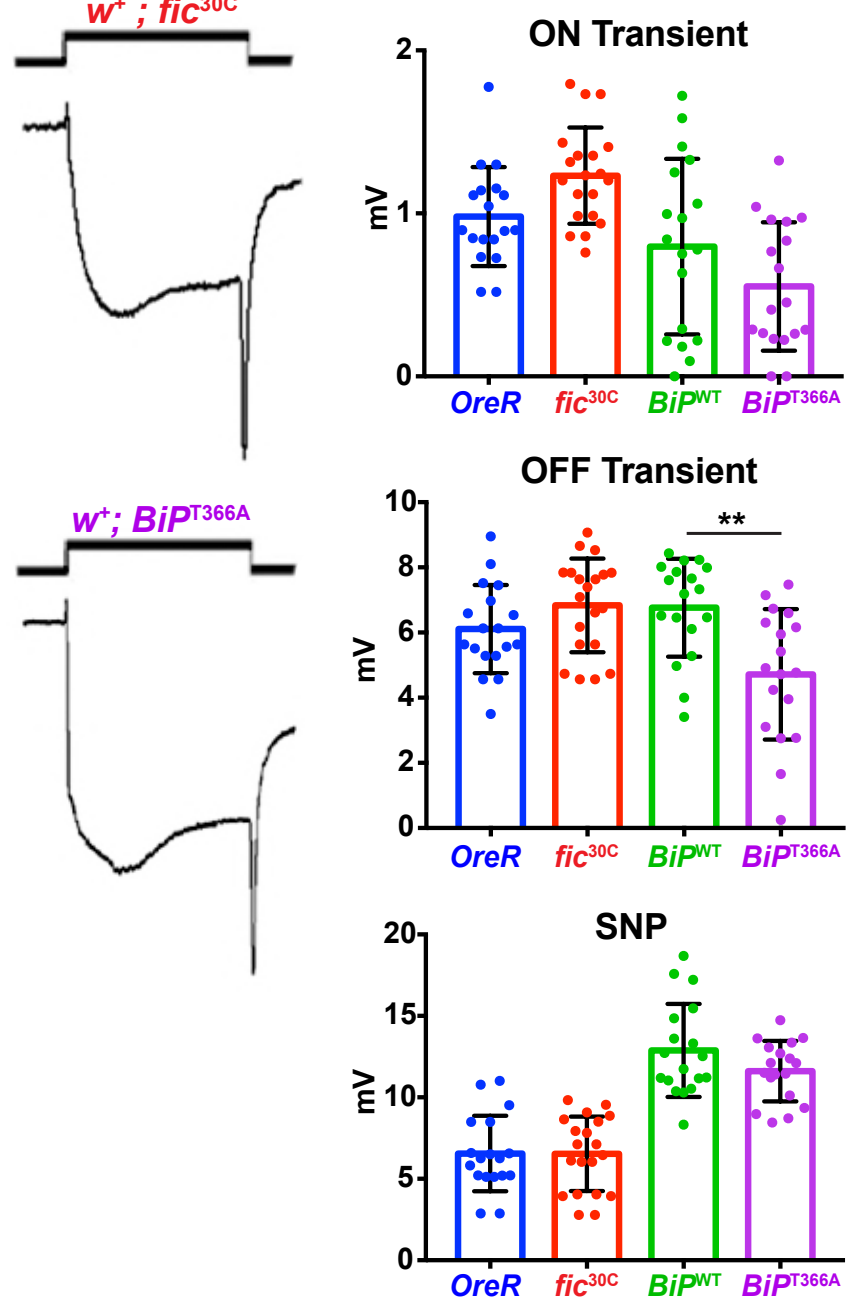

Figure 1- figure supplement 3. Red eye pigment suppresses ERG phenotypes of fic $^{30 \mathrm{C}}$ and BiP $^{\mathrm{T} 366 \mathrm{~A}}$ mutants.

(A) ERGs of OreR and red-eyed $f_{i c}^{30 \mathrm{C}}$ flies as well as $\mathrm{BiP}^{\mathrm{WT}}$ and $\mathrm{BiP}^{\mathrm{T} 366 \mathrm{~A}}$ animals. (B)

780 Quantification of ERG data. Bar graphs show means +/- SD. **, $\mathrm{p}<0.01, \mathrm{n}=18$ flies per 781 genotype and condition. 


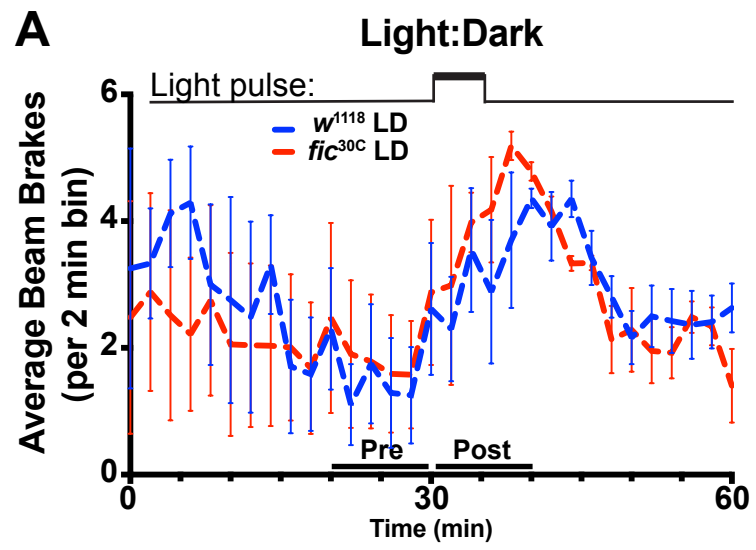

B

Light:Light

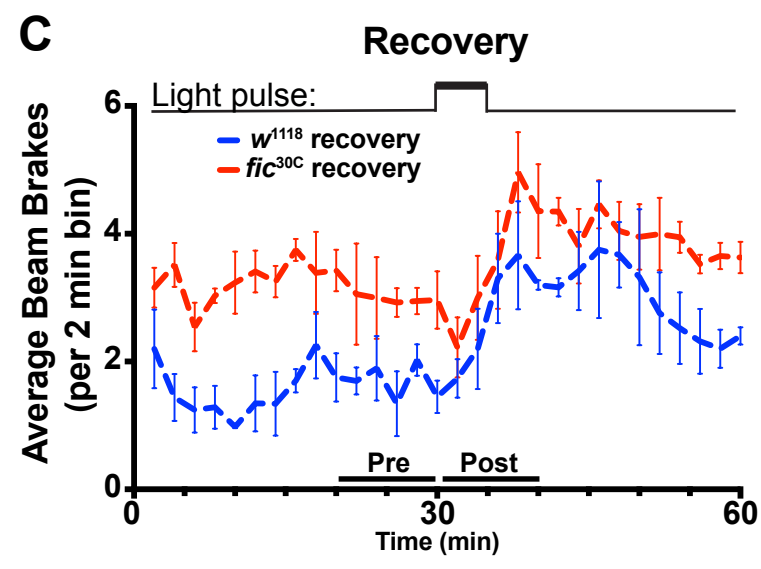

D Change in Activity
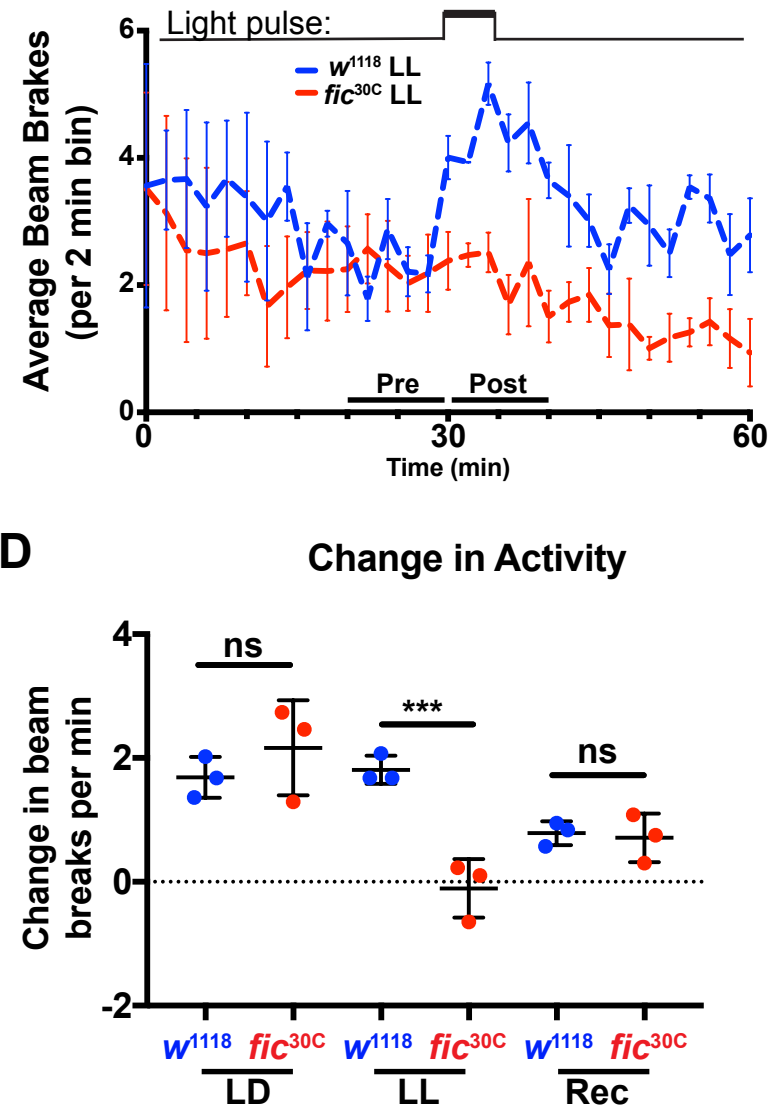

Figure 3- figure supplement 1. Light-induced defects in light-startle activity in fic ${ }^{30 \mathrm{C}}$ mutants.

(A, B, \& C) Actogram of $w^{1118}$ or $f i c^{30 \mathrm{C}}$ flies reared in LD for three days (A), LL for three days (B), or recovery condition (three days in LL then three days in LD) (C). Light pulse is indicated by upper bars. Data is averaged from three biological replicas, each containing 16 flies per genotype. Data were collected in two-minute bins. Error bars represent Standard Error. (D) Quantification of change in beam breaks per 2-min bin for the $10 \mathrm{~min}$ intervals before and after the onset of the light pulse in each experiment. Bar graphs show means +/- SD. ***, $p<0.01, \mathrm{n}=$ 3 experimental repeats with 16 flies per genotype and condition. Dead flies and those with a change in activity greater than 3 deviations from the median were excluded. 


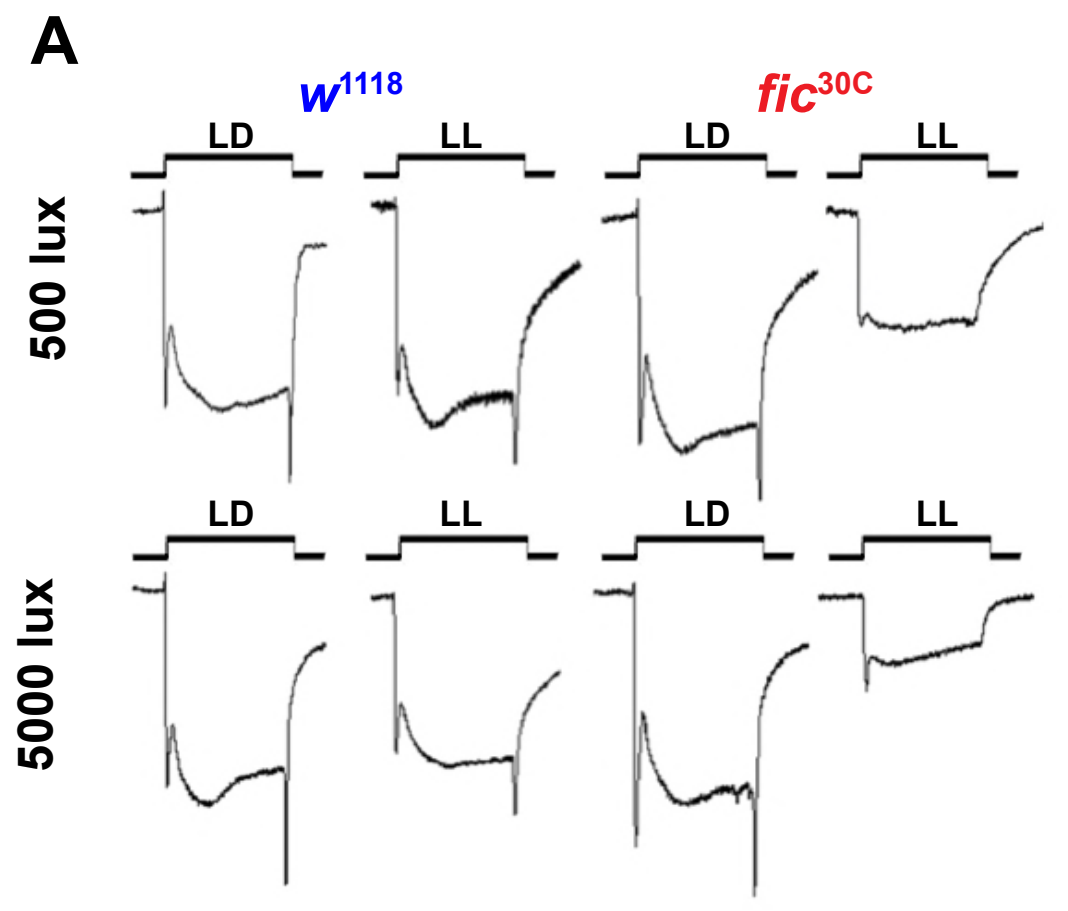

B

ON Transient
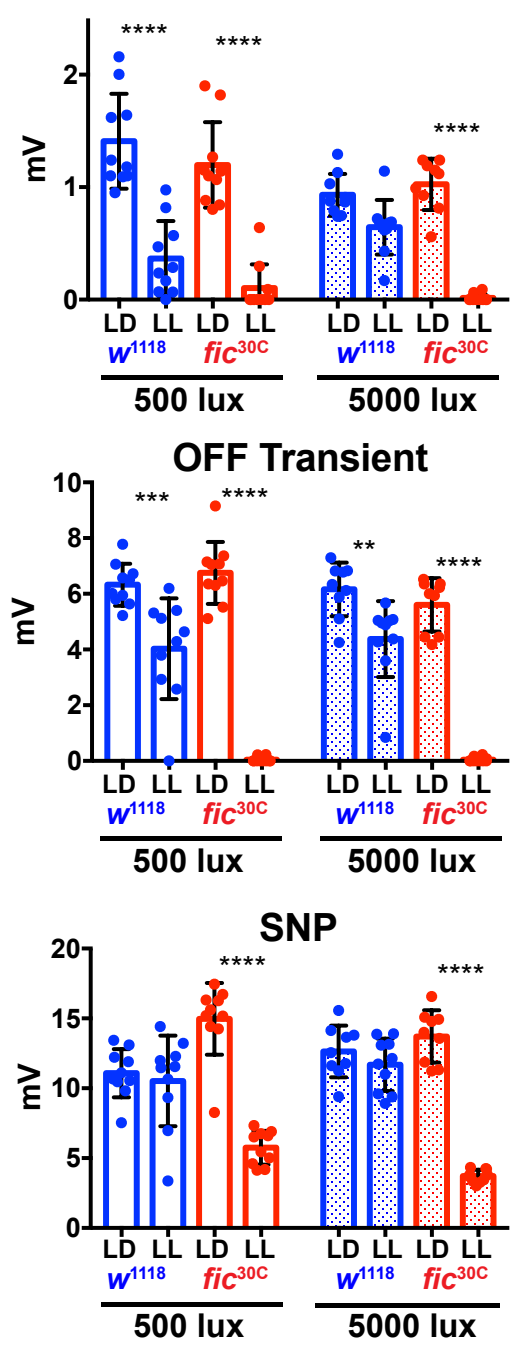

Figure 3- figure supplement 2. fic $^{30 \mathrm{C}}$ mutants are sensitive to constant light, regardless of total intensity.

(A) Representative ERGs of flies following 3 days of LL or LD with either 500 lux or 5000 lux

797 light, showing $f \mathrm{c}^{30 \mathrm{C}}$ null animals lose ON/OFF transients and have reduced SNPs with constant 798 light, regardless of intensity, but under LD conditions, even at 5000 lux, have healthy ERG responses. (B) Quantification of ERG data. Bar graphs show means +/- SD. ****, p $<0.0001$; ***, $\mathrm{p}<0.001 ; *, \mathrm{p}<0.05 ; \mathrm{n}=10$ flies per genotype and condition. 

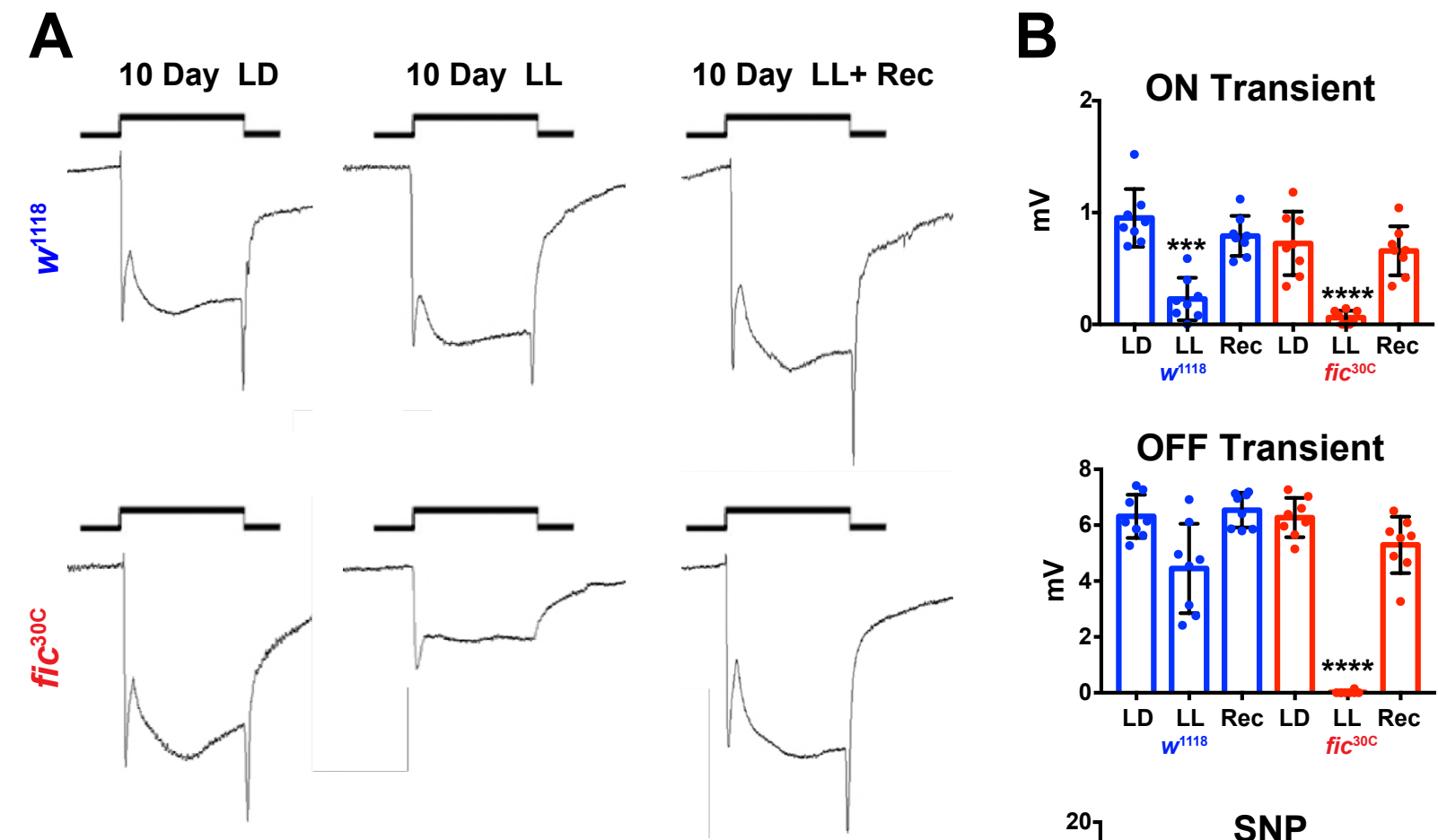
days of $\mathbf{L L}$.

(A) Representative ERGs of flies following 10 days of LL, 10 days LD (500 lux), and 3 days Recovery following 10 days. (B) Quantification of ERG data. Bar graphs show means +/- SD. $* * * *, \mathrm{p}<0.0001 ; * * *, \mathrm{p}<0.001 ; *, \mathrm{p}<0.05 ; \mathrm{n}=8$ flies per genotype and condition. 


\begin{tabular}{|c|c|c|c|c|c|c|c|c|c|}
\hline \multirow{2}{*}{ Line } & \multirow{2}{*}{$\begin{array}{l}\text { RNAi } \\
\text { Target } \\
\text { Gene }\end{array}$} & \multicolumn{5}{|c|}{ Eye Roughness Score } & \multirow{2}{*}{$\begin{array}{l}\text { Weighted } \\
\text { Average }\end{array}$} & \multirow{2}{*}{$\begin{array}{l}\text { Number } \\
\text { of Flies }\end{array}$} & \multirow{2}{*}{$\begin{array}{l}\text { P-Value } \\
\text { (Fisher's) }\end{array}$} \\
\hline & & 0 & 1 & 2 & 3 & 4 & & & \\
\hline Control & None & 0 & 1 & 68 & 3 & 0 & 2.03 & 72 & $\mathrm{n} / \mathrm{a}$ \\
\hline BS36815 & 4EBP & 0 & 0 & 96 & 0 & 0 & 2.00 & 96 & 0.032 \\
\hline v2935 & ATF4 & Lethal & Lethal & Lethal & Lethal & Lethal & Lethal & Lethal & Lethal \\
\hline BS25985 & ATF4 & 0 & 0 & 51 & 98 & 0 & 2.66 & 149 & $2.20 \mathrm{E}-16$ \\
\hline BS26211 & ATF6 & 0 & 2 & 68 & 2 & 0 & 2.00 & 72 & 1.000 \\
\hline BS64873 & CaBP1 & 0 & 3 & 42 & 0 & 0 & 1.93 & 45 & 0.137 \\
\hline BS58172 & Calnexin & 0 & 2 & 41 & 1 & 0 & 1.98 & 44 & 0.688 \\
\hline v7799 & elF2a & 0 & 2 & 54 & 0 & 0 & 1.96 & 56 & 0.302 \\
\hline v104562 & elF2a & 0 & 0 & 48 & 0 & 0 & 2.00 & 48 & 0.388 \\
\hline BS55657 & ergic53 & 0 & 5 & 35 & 7 & 0 & 2.04 & 47 & 0.007 \\
\hline BS35023 & Gadd45 & 0 & 0 & 45 & 3 & 0 & 2.06 & 48 & 0.811 \\
\hline BS34346 & GP93 & 0 & 0 & 33 & 5 & 0 & 2.13 & 38 & 0.122 \\
\hline v39561 & Ire1 & 0 & 0 & 33 & 40 & 0 & 2.55 & 73 & $4.14 \mathrm{E}-12$ \\
\hline v39562 & Ire1 & 0 & 0 & 15 & 47 & 0 & 2.76 & 62 & $2.20 \mathrm{E}-16$ \\
\hline BS62156 & Ire1 & 0 & 0 & 0 & 57 & 2 & 3.03 & 59 & $2.20 \mathrm{E}-16$ \\
\hline BS36743 & Ire1 & 0 & 0 & 0 & 14 & 13 & 3.48 & 27 & $2.20 \mathrm{E}-16$ \\
\hline BS28039 & PDI & 0 & 0 & 20 & 2 & 0 & 2.09 & 22 & 0.685 \\
\hline v110278 & PERK & 0 & 0 & 3 & 45 & 4 & 3.02 & 52 & $2.20 \mathrm{E}-16$ \\
\hline v16427 & PERK & 0 & 0 & 0 & 13 & 30 & 3.70 & 43 & $2.20 \mathrm{E}-16$ \\
\hline BS35162 & PERK & 0 & 0 & 28 & 43 & 0 & 2.61 & 71 & $6.84 \mathrm{E}-14$ \\
\hline BS42499 & PERK & 0 & 0 & 26 & 65 & 50 & 3.17 & 141 & $2.20 \mathrm{E}-16$ \\
\hline BS36755 & Xbp1 & Lethal & Lethal & Lethal & Lethal & Lethal & Lethal & Lethal & Lethal \\
\hline BS25990 & Xbp1 & Lethal & Lethal & Lethal & Lethal & Lethal & Lethal & Lethal & Lethal \\
\hline
\end{tabular}

\section{Table 1. Genetic interactions between Fic and UPR genes.}

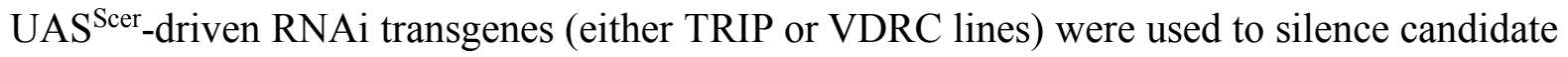
UPR and ER stress-related genes in a $\mathrm{fic}^{30 \mathrm{C}} /+$ heterozygous background, with either LongGMRGal4, UAS-Fic ${ }^{\mathrm{E} 247 \mathrm{G}}$ or LongGMR-Gal4 only. Eye roughness was scored for individual flies and averaged for each cross. Table reports number of flies scored in each group $(0=$ no roughness, $2=$ mildly rough (control flies), $4=$ severely rough, 1 and 3 are intermediate phenotypes) and the weighted average of the eye roughness. Significance differences are highlighted in yellow, and p-value's were determined using Fisher's Exact Test for categorical data, comparing the effects of each gene knockdown with the control group (top line, fic ${ }^{30 \mathrm{C} /+}$; LongGMR-Gal4, UAS$\left.\mathrm{Fic}^{\mathrm{E} 247 \mathrm{G}}\right)$. Interactions were considered significant for any individual test if $p<0.003$ as determined using Bonferroni’s multiple comparison adjustment. 